\title{
Analysis of Power System Oscillations for Developing Synchrophasor Data Applications
}

\author{
Luigi Vanfretti \\ Royal Institute of Technology (KTH) \\ Stockholm, Sweden \\ luigi.vanfretti@kth.se
}

\author{
Joe H. Chow \\ Rensselaer Polytechnic Institute \\ Troy, NY, USA \\ chowjorpi.edu
}

\begin{abstract}
Electromechanical modes are predominantly determined by the machine rotor angles and speeds, and as a result, they provide the best visibility of such modes. These electromechanical modes are also observable in the network variables such as voltages and line currents, which are measured by PMUs. In this paper, by analyzing the electromechanical modes in the network variables, we can trace the propagation of electromechanical oscillations in the power network following a disturbance. In large power networks with multiple transfer paths across the interconnection it is not always clear how and where the oscillations propagate. Here, we seek to provide a rationale explaining how (the qualitative behavior) and where (transmission lines) network oscillations propagate. We first analyze the oscillations from real PMU data originating from a system-wide disturbance. Applying eigenvalue and sensitivity analysis we provide an analytical framework to understand the nature of the network oscillations. Closed-form expressions for the network sensitivities are provided. The results here important for building reduced models to assess transient stability and the selection of network variables as input signals to damp inter-area mode oscillations.
\end{abstract}

\section{Keywords}

Inter-area oscillation, network oscillations, modal analysis, linear analysis techniques, synchronized phasor measurements, inter-area mode tracing

\section{Introduction}

Inter-area modes are predominantly determined by the machine rotor angles and speeds [1]- [5], and as a result, they provide the best visibility of such modes. Thus electromechanical modes are also observable in the network variables such as voltages and line currents, which are measured by PMUs [6] and observed through simulations [7]. In this paper, by computing the electromechanical modes in the network variables, we can trace the propagation of electromechanical oscillations in the power network following a disturbance. These network variables have received less attention because they are algebraic variables which can change abruptly. Recently, an analytical framework to study voltage inter-area oscillations present in the bus voltage and frequency variables has been proposed [8]. This technique uses the linearized model of a power system with bus voltages and frequencies as output variables.

The purpose of this investigation is to generalize the results in [8] to analyze power system oscillations which may be present in any type of network variables. To motivate, we first analyze the oscillations observed during a major system disturbance that took place on $2 / 26 / 2008$ in the Florida Reliability Coordination Council (FRCC) service area $[9,10]$. The PMU measurements show that after the initial transient, the oscillations consist mainly of one single-frequency mode, peaking at different instants. A question of interest is whether these oscillations originate from a single mode. If this is so, how should the time delay be explained.

With the goal of explaining the origin of the phase shifts in the network variables, we use the multi-machine linearized power system electromechanical model [11] and extend the mode shape concepts to the network variables. We show that with no damping and constant impedance loads, all electromechanical oscillations are in phase. However, when damping or control equipment such as voltage regulator models are included, the eigenvector matrix will indicate phase shifts. The time delays related to these phase shifts show a strong resemblance to those observed in PMU data.

To obtain the mode shapes, we perform a detailed sensitivity analysis of the network variables and provide analytical expressions [12]. This analysis provides a theoretical understanding of oscillations as directly measured by PMUs on high-voltage transmission systems. Finally, network sensitivities and eigenvectors are used to obtain the modal components in the network variables, thus providing a rationale explaining the phase shifts observed in the modal components of the synchrophasor data.

The remainder of the paper is organized as follows. In Section 2 we analyze the network oscillations originating from the $2 / 26 / 2008$ FRCC disturbance. In Section 3 we determine the origin of the phase shifts in the electromechanical mode shape. In Section 4 we perform sensitivity analysis of the network variables and provide closed-form expressions of the network sensitivities. Afterwards, in Section 5 we map the network sensitivities onto the electromechanical 


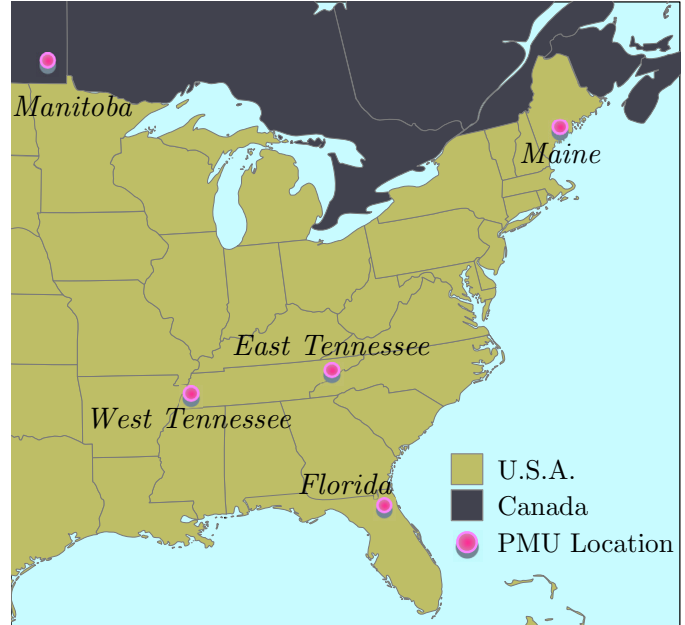

(a) PMU Locations

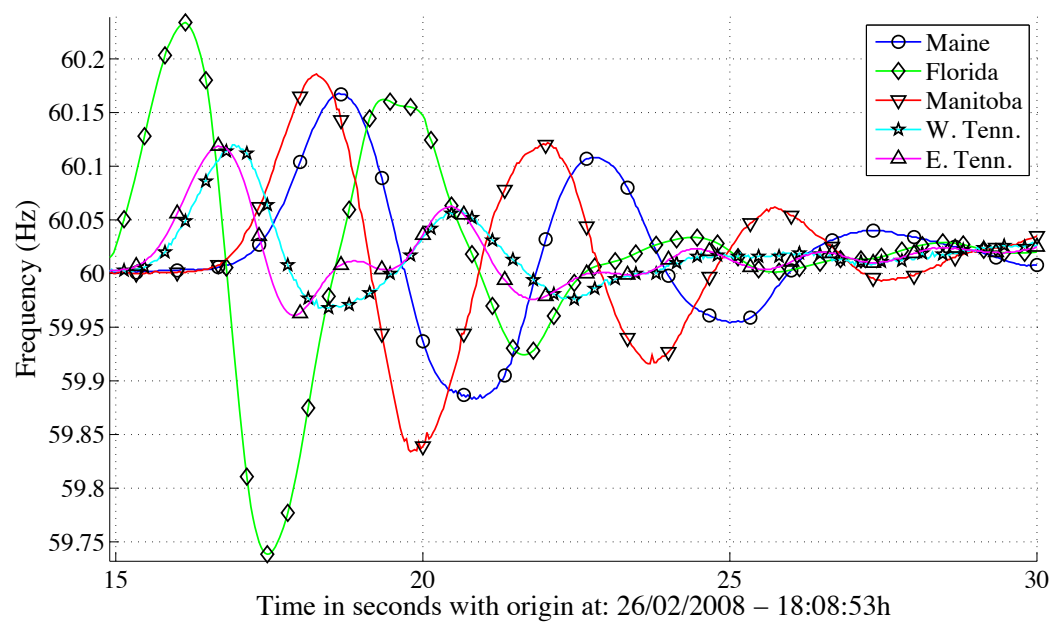

(b) Bus Frequency

Figure 1: PMU locations and recorded measurements during the 2008 Florida Disturbance

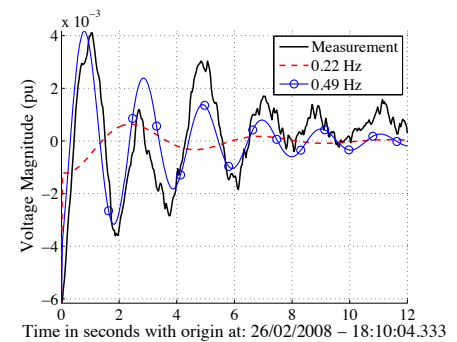

(a) Voltage Magnitude

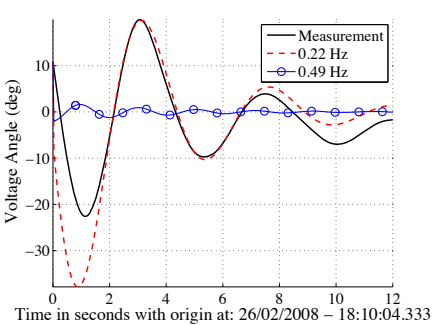

(b) Voltage Angle
Figure 2: Identified Oscillatory Components in the Voltage Phasor at W. Tenn.

mode shapes and explain the nature of the modal components in the network variables, illustrating with a fourgenerator, two-area system $[12,13]$. Applications for interarea mode tracing and PMU siting are suggested in Section 6 and conclusions are presented in Section 7.

\section{Nature of Network Oscillations Observed from Phasor Measurement Data}

We study the power system inter-area oscillations excited by the FRCC system disturbance by analyzing archived phasor measurement data. The PMUs considered in this analysis are Manitoba, near the city of Winnipeg, Canada. Main near Bangor, Maine. Florida near Jacksonville, Florida. West Tennessee (W. Tenn.), near Memphis, Tennessee, and East Tennessee (E. Tenn.), near Knoxville, Tennessee, as shown in Fig. 1a. In Fig. 1b we plot the bus frequency measured during the disturbance by the PMUs, showing the wide-area impact of the disturbance. The steady state frequency deviation is approximately $\Delta f=30$ $\mathrm{mHz}$, while the electromechanical swing is propagated from Florida, to E. Tenn. and W. Tenn., and subsequently to Manitoba, and finally Maine.

We aim to analyze the oscillatory components and characteristics contained in these measurements. We use the

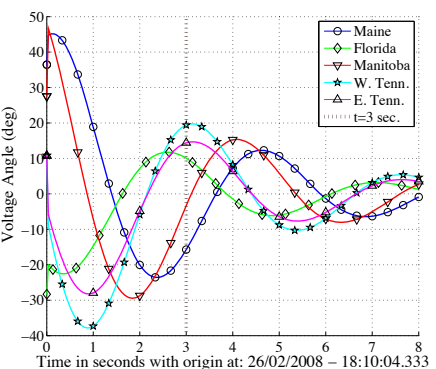

(a) $\Delta \theta_{i} 0.22 \mathrm{~Hz}$ Components

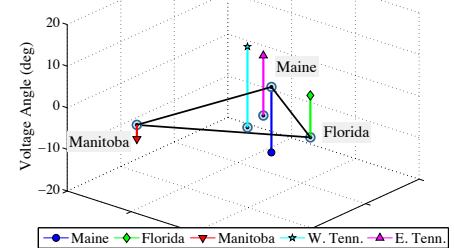

(c) $0.22 \mathrm{~Hz} \Delta \theta_{i}$ projection

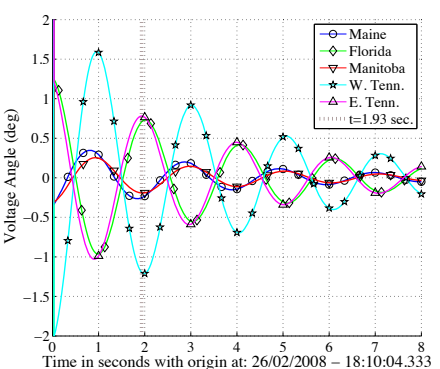

(b) $\Delta \theta_{i} 0.49 \mathrm{~Hz}$ Components
Figure 3: Voltage Angle Inter-area Oscillations and Projections

Eigensystem Realization Algorithm (ERA) [14] to identify the individual modal components in the voltage magnitude, voltage angle, and active power flow measurements available from each PMU. The identified low-frequency interarea modes include frequencies of $0.22 \mathrm{~Hz}$ and $0.49 \mathrm{~Hz}$. In Fig. 2 the voltage phasors measurements of the PMU at W. Tenn. are shown along with their ERA approximation for the 0.22 and $0.49 \mathrm{~Hz}$ components of the signals.

All identified components for the $0.22 \mathrm{~Hz}$ mode in the voltage angle are shown in Fig. 3a, and those for the $0.49 \mathrm{~Hz}$ mode are shown in Fig. 3b. A snapshot of the voltage angle components is taken at $t=3 \mathrm{sec}$, and is used for the projection of the $0.22 \mathrm{~Hz}$ mode shown in Fig. 3c. For the $0.49 \mathrm{~Hz}$ mode a snapshot of the voltage angle components is taken at $t=1.93 \mathrm{sec}$, and used for the projection in Fig 
3 d. The starting time $t=0 \mathrm{sec}$ corresponds to $18: 10: 04.333$ hrs. From the voltage angles of the $0.22 \mathrm{~Hz}$ mode we note that Florida oscillates against Maine and Manitoba, that is, it is a North vs South mode.

The $0.49 \mathrm{~Hz}$ mode is more difficult to analyze from this limited data set. However, it can be noted that the voltage angle at W. Tenn., E. Tenn., and Florida have the largest oscillations while Manitoba and Maine have a less significant contribution. More important, W. Tenn. and E. Tenn. are in anti-phase suggesting that the pivot node of the oscillation is located somewhere between them.

The most important observation that could be made about the oscillations discussed above is the following: for all of the network variables, the independent modal components do not peak at the same instants. There is in fact a time delay between the modal components. This time delay can be viewed as a phase shift between the modal components in the frequency domain. In the next section we will investigate this phase shift by analyzing the mode shapes of a test network.

\section{The Electromechanical Mode Shape}

\subsection{Multi-Machine Electromechanical Model}

In this section we develop a basic understanding on the origin of electromechanical mode phase shifts by performing eigenanalysis on different linearized models of multi machine power systems. We start with the electromechanical model [15]. For an $N$-machine power system, the linearized electromechanical model in state-space form is given by

$$
\underbrace{\left[\begin{array}{c}
\Delta \dot{\delta} \\
\Delta \dot{\omega}
\end{array}\right]}_{\dot{x}}=\underbrace{\left[\begin{array}{cc}
0 & \Omega \boldsymbol{I}_{(N \times N)} \\
M^{-1} K & M^{-1} \boldsymbol{D}
\end{array}\right]}_{\bar{A}} \underbrace{\left[\begin{array}{c}
\Delta \delta \\
\Delta \omega
\end{array}\right]}_{\boldsymbol{x}}
$$

where

$$
\begin{gathered}
\Delta \boldsymbol{\delta}=\left[\begin{array}{lll}
\Delta \delta_{1} & \ldots \Delta \delta_{N}
\end{array}\right]^{T},(N \times 1) \\
\boldsymbol{\Delta} \boldsymbol{\omega}=\left[\begin{array}{lll}
\Delta \omega_{1} & \ldots \Delta \omega_{N}
\end{array}\right]^{T},(N \times 1) \\
\boldsymbol{M}^{-1}=\operatorname{diag}\left(\frac{1}{2 H_{i}}\right),(N \times N) \\
\boldsymbol{D}=\operatorname{diag}\left(D_{i}\right),(N \times N) ; \boldsymbol{K}=\left[K_{i j}\right],(N \times N)
\end{gathered}
$$

where $i, j=1, \ldots, N$. We will refer to this model (1) as the electromechanical model with damping, and to matrix $\overline{\boldsymbol{A}}$ as the state matrix with damping. If the damping terms are neglected $\left(D_{i} \approx 0\right)$ the model becomes

$$
\underbrace{\left[\begin{array}{c}
\Delta \dot{\boldsymbol{\delta}} \\
\Delta \dot{\boldsymbol{\omega}}
\end{array}\right]}_{\dot{\boldsymbol{x}}}=\underbrace{\left[\begin{array}{cc}
\mathbf{0} & \Omega \boldsymbol{I}_{(N \times N)} \\
\boldsymbol{A}_{\omega} & \mathbf{0}
\end{array}\right]}_{\boldsymbol{A}_{\omega}=\boldsymbol{M}^{-1} \boldsymbol{K}} \underbrace{\left[\begin{array}{c}
\Delta \boldsymbol{\delta} \\
\Delta \omega
\end{array}\right]}_{\boldsymbol{x}}
$$

We will refer to $\boldsymbol{A}$ as the state matrix without damping. Many properties of $\overline{\boldsymbol{A}}$ and $\boldsymbol{A}$ have been studied $[11,15,16]$. In this investigation some characteristics of the eigenvectors of $\boldsymbol{A}$ and $\overline{\boldsymbol{A}}$ are analyzed. These characteristics have strong effects on the phase shift of network variables as discussed later.

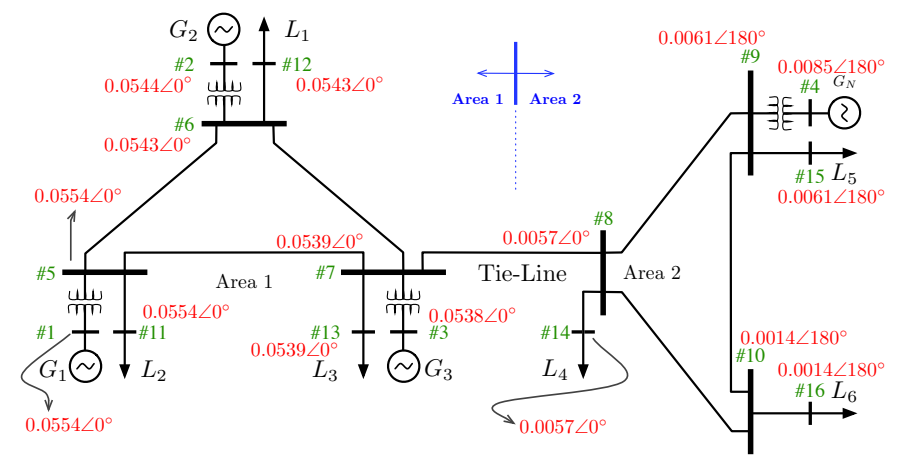

Figure 4: Two-Area Four-Machine Power System and Voltage Magnitude Mode Shape for the Inter-area Mode (No Damping)

\subsection{Eigenvectors of the Electromechanical Models}

Consider the two-area four-machine power system shown in Fig. $4[12,13]$. The system is modeled using (3), linearized about an equilibrium. In the resulting state matrix $\boldsymbol{A}$ all elements corresponding to the machine damping $\boldsymbol{M}^{-1} \boldsymbol{D}$ are zero. Eigenanalysis is performed on $\boldsymbol{A}$ resulting in the eigenvector matrix

$$
\boldsymbol{W}(\boldsymbol{A})=\left[\begin{array}{cccc}
0.5 & -0.5930 & 0.7871 & -0.0547 \\
0.5 & -0.5997 & -0.6167 & -0.0512 \\
0.5 & -0.4539 & 0.0155 & 0.9972 \\
0.5 & 0.2876 & -0.0015 & -0.0041
\end{array}\right]
$$

Note that only the components corresponding to the machine angles are shown. All the components of $\boldsymbol{W}(\boldsymbol{A})$ are real. Column 2, the inter-area mode mode shape in Fig. 5a, shows that $G_{1}, G_{2}$ and $G_{3}$ are oscillating against $G_{4}$, that is, Area 1 oscillates against Area 2.

Column 3, the mode shape for Local Mode 1 in Fig. 5b, indicates that $G_{1}$ and $G_{2}$ are mostly oscillating against each other. Finally, Local Mode 2 (Column 4) is mostly confined within Area 1 with $G_{3}$ oscillating against $G_{1}$ and $G_{2}$. The most important characteristic to note is that the oscillations are either completely in-phase or anti-phase as reflected by the time-responses in Fig. 5c for the inter-area mode and Fig. 5d for Local Mode 1.

Using (1) we analyze the effect that machine damping has on the eigenvectors. The elements corresponding to the machine damping $\boldsymbol{M}^{-1} \boldsymbol{D}$ in $\overline{\boldsymbol{A}}$ are given by

$$
\boldsymbol{M}^{-1} \boldsymbol{D}=\operatorname{diag}\left(\left[\begin{array}{llll}
-0.001 & -0.3 & -0.015 & -0.2
\end{array}\right]\right)
$$

and all other elements remain unchanged with respect to A.

Eigenanalysis is performed on $\bar{A}$, yielding the eigenvector matrix in (6) at the top of p. 4. Note that the eigenvector matrix is now complex. For convenience it is shown in polar form. The main oscillatory characteristics discussed for the mode shapes from $\boldsymbol{A}$ are maintained for the mode shapes of $\overline{\boldsymbol{A}}$. However, the components of each mode shape now present a phase shift due to the inclusion of machine damping. This phase shift is readily observed in the phasor 


$$
\boldsymbol{W}(\overline{\boldsymbol{A}})=\left[\begin{array}{cccc}
0.5 \angle 0^{\circ} & 0.5882 \angle 172.6196^{\circ} & 0.7878 \angle 0^{\circ} & 0.0548 \angle-178.5539^{\circ} \\
0.5 \angle 0^{\circ} & 0.6066 \angle-180^{\circ} & 0.6084 \angle 165.8682^{\circ} & 0.0507 \angle 172.6111^{\circ} \\
0.5 \angle 0^{\circ} & 0.4548 \angle 176.1869^{\circ} & 0.0951 \angle 68.6262^{\circ} & 0.9972 \angle 0^{\circ} \\
0.5 \angle 0^{\circ} & 0.2814 \angle-6.3759^{\circ} & 0.0072 \angle-116.0791^{\circ} & 0.0041 \angle 176.6467^{\circ}
\end{array}\right]
$$

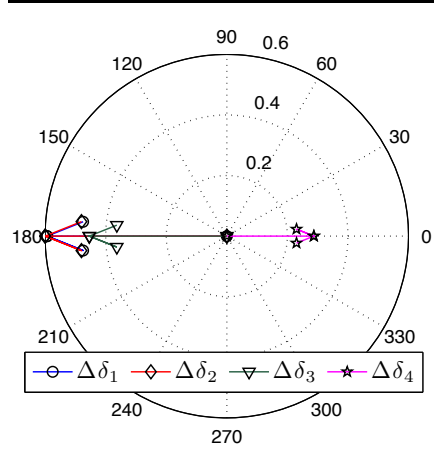

(a) Inter-area Mode

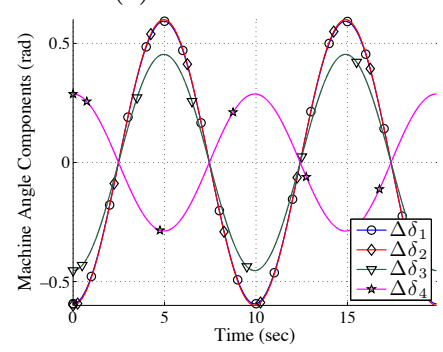

(c) Inter-area Mode

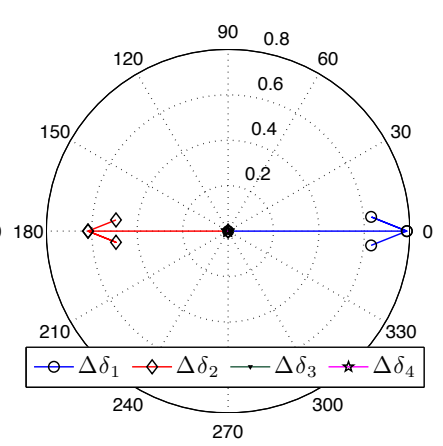

(b) Local Mode 1

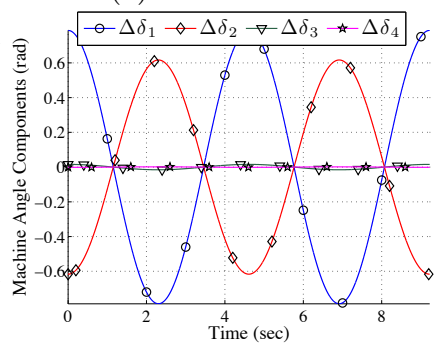

(d) Local Mode 1

Figure 5: Machine angle mode shapes (no damping), phasor diagram (a) and (b), and time response (c) and (d)

diagrams in Figs. 6a and 6b. A more important observation is that in the time domain these phase shifts translate to time delays, making the oscillations in each mode peak at different instants as shown in Figs. $6 \mathrm{c}$ and $6 \mathrm{~d}$. It is interesting to note that the time-shifts shown in Figs. $6 \mathrm{c}$ and $6 \mathrm{~d}$ strongly resembles those of the measurement data presented in the previous section. The phase shifts are particularly exhibited for "non-dominant" bus locations. Why do the network measurements exhibit phase shifts similar to those in the machine angle mode shapes of $\overline{\boldsymbol{A}}$ ? In the following section we build upon the understanding gained from the mode shapes from $\overline{\boldsymbol{A}}$, and generalize the results obtained in [8].

\section{Sensitivity Analysis of Network Variables}

\subsection{Network Sensitivities}

By computing the sensitivities of the entire network, it is possible to predict the incremental behavior of the network variables when a small perturbation occurs in the power system. This is of particular interest because PMUs are capable of measuring synchronized network variable changes across wide-areas of the power system that emerge from small perturbations. Thus, an understanding of network sensitivities can provide insight for PMU data analysis of small signal oscillations occurring in large scale power networks.

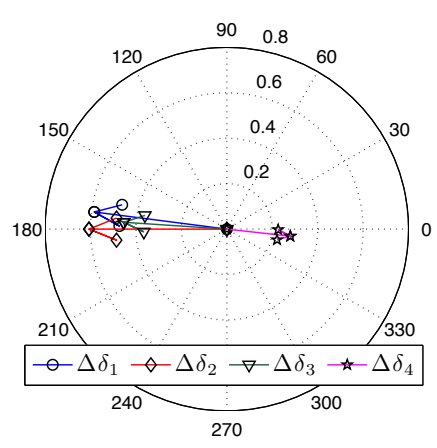

(a) Inter-area Mode

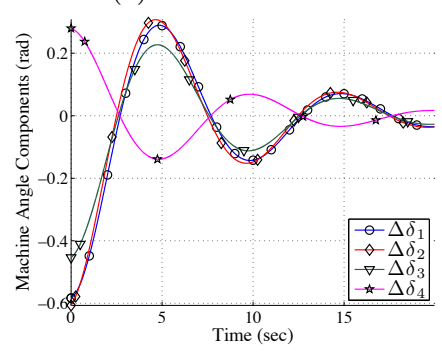

(c) Inter-area Mode

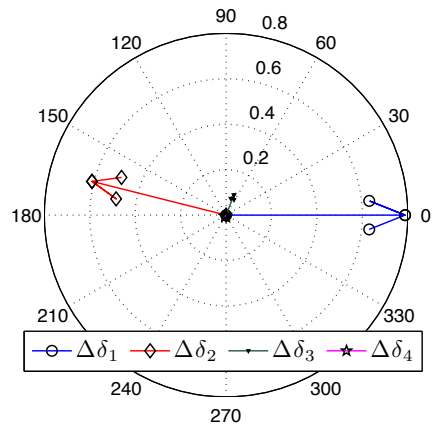

(b) Local Mode 1

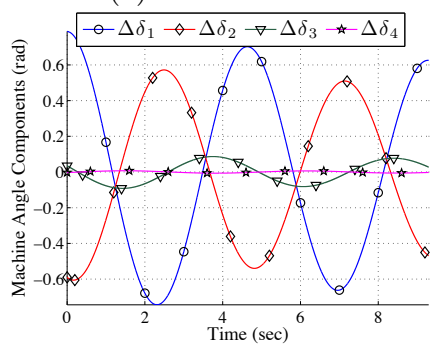

(d) Local Mode 1
Figure 6: Machine angle mode shapes (damping), phasor diagram (a) and (b), and time response (c) and (d)

To illustrate, consider the $n$-bus, $N$-machine power system shown in Fig. 7. Buses $i$ to $(i+4)$ are transmission and load buses remotely connected to generators $j=1, \ldots, N$. Regardless of their distance from the generators, these buses will be affected by changes in the internal machine angle of any generator. For example, a change in the internal angle of machine $j, \Delta \delta_{j}$, will be reflected in the voltage magnitude at the $i$-th bus, $V_{i}$, in a proportion dictated by the network sensitivity $\left.\left(\partial V_{i} / \partial \delta_{j}\right)\right|_{(\text {o) }}$. The total change in the voltage magnitude at bus $i, \Delta V_{i}$, will be the sum of all the changes in the machine internal angles scaled by their corresponding network sensitivity.

Similarly, if the current flow among buses $i$ to $(i+4)$ is as indicated in Fig. 7, the change in the current flow will also be affected by the change in machine internal angles. The complex line current flow changes will be proportionally distributed satisfying Kirchhoff's current law, i.e., $\Delta \tilde{I}_{i(i+1)}=\Delta \tilde{I}_{(i+1)(i+2)}+\Delta \tilde{I}_{(i+1)(i+3)}+\Delta \tilde{I}_{(i+1)(i+4)}$. Thus, it becomes possible to trace how the current oscillations are being divided among multiple lines/paths and propagated across the entire network.

To obtain the change of the voltage magnitude $\left(\Delta V_{i}\right)$ and angle $\left(\Delta \theta_{i}\right)$ at the $i$-th bus of the network with respect to the change of machine internal angles we obtain the Taylor series expansion of $V_{i}$ and $\theta_{i}$ about an equilibrium. Denoting $N$ as the total number of machines in the system, $n$ 


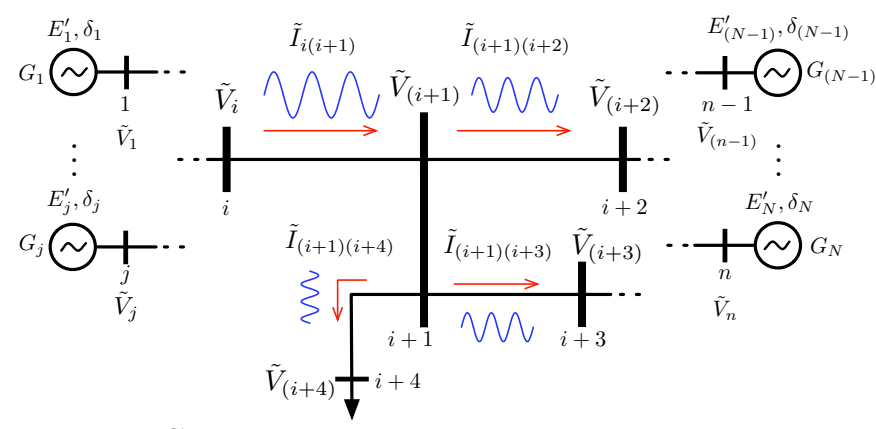

Figure 7: Changes in the network variables with respect to changes in $\delta_{j}$ 's

the total number of buses in the system, and ignoring the higher order terms, the change of voltage magnitude at bus $i$ due to the change in the machine internal angles is given by

$$
\begin{aligned}
\Delta V_{i}= & \left.\left(\frac{\partial V_{i}}{\partial \delta_{1}}\right)\right|_{0} \Delta \delta_{1}+\left.\left(\frac{\partial V_{i}}{\partial \delta_{2}}\right)\right|_{0} \Delta \delta_{2}+\ldots \\
& +\left.\left(\frac{\partial V_{i}}{\partial \delta_{n}}\right)\right|_{0} \Delta \delta_{n}
\end{aligned}
$$

where $\left.\left(\partial V_{i} / \partial \delta_{j}\right)\right|_{0}$ is the sensitivity of the $i$-th bus voltage magnitude to the $j$-th machine angle at the equilibrium point. In matrix form, the voltage magnitude changes at buses $i=1, \ldots, n$ due to the change in the machine internal angles are given by

$$
\underbrace{\left[\begin{array}{c}
\Delta V_{1} \\
\vdots \\
\Delta V_{n}
\end{array}\right]}_{\boldsymbol{\Delta} \boldsymbol{V}}=\underbrace{\left[\begin{array}{ccc}
\left.\left(\frac{\partial V_{1}}{\partial \delta_{1}}\right)\right|_{(0)} & \cdots & \left.\left(\frac{\partial V_{1}}{\partial \delta_{j}}\right)\right|_{(0)} \\
\vdots & \ddots & \vdots \\
\left.\left(\frac{\partial V_{n}}{\partial \delta_{1}}\right)\right|_{(0)} & \cdots & \left.\left(\frac{\partial V_{n}}{\partial \delta_{j}}\right)\right|_{(0)}
\end{array}\right]}_{\boldsymbol{C}_{\mathrm{V} \delta}} \underbrace{\left[\begin{array}{c}
\Delta \delta_{1} \\
\vdots \\
\Delta \delta_{n}
\end{array}\right]}_{\boldsymbol{\Delta} \boldsymbol{\delta}}
$$

where $\boldsymbol{\Delta} \boldsymbol{V}$ is the vector of bus voltage magnitude changes of size $(n \times 1)$, and $\boldsymbol{C}_{\mathrm{V} \delta}$ is the bus voltage magnitude sensitivity matrix of size $(n \times N)$.

Sensitivities may also be obtained for any other network variable can be obtained similarly. For the bus voltage angle changes, we have

$$
\Delta \boldsymbol{\theta}=\boldsymbol{C}_{\theta \delta} \boldsymbol{\Delta} \delta
$$

where $\boldsymbol{C}_{\theta \delta}$ is the bus voltage angle sensitivity matrix of size $(n \times N)$.

In a power system, with a total number of $\ell$ transmission lines connecting the sending end buses, $f$, to the receiving end buses, $t$, the current magnitude and angle changes w.r.t. the machine angle changes are given by

$$
\Delta \boldsymbol{I}_{\mathrm{ft}}=\boldsymbol{C}_{\mathrm{I}_{\mathrm{ft}} \delta} \boldsymbol{\Delta} \boldsymbol{\delta} \quad(10) \quad \Delta \phi_{\mathrm{ft}}=C_{\phi_{\mathrm{ft}} \delta} \boldsymbol{\Delta} \boldsymbol{\delta}
$$

where $\boldsymbol{\Delta} \boldsymbol{I}_{\mathrm{ft}}$ and $\boldsymbol{\Delta} \boldsymbol{\phi}_{\mathrm{ft}}$ are the current magnitude and angle changes, respectively. Matrix $\boldsymbol{C}_{\mathrm{I}_{\mathrm{ft}} \delta}$ is the current magnitude sensitivity matrix while $\boldsymbol{C}_{\phi_{\mathrm{ft}} \delta}$ is the current angle sensitivity matrix, both of size $(\ell \times N)$.

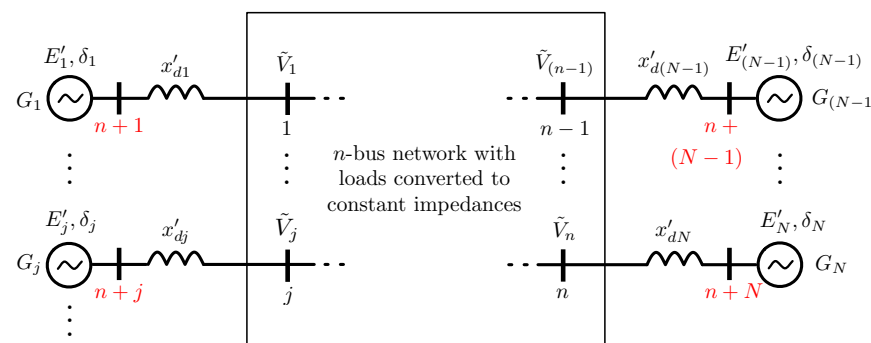

Figure 8: $n$-bus network extended to the machine internal nodes

In general, the sensitivity matrices $\boldsymbol{C}_{\mathrm{V} \delta}$ and $\boldsymbol{C}_{\theta \delta}$ are obtained by numerical perturbation using simulation software such as PST [17]. Analytical expressions may provide further insight on their properties and the parameters affecting them. In the next section we provide closed form expressions of different network sensitivities.

\subsection{Analytical Derivation of the Network Sensitivities}

The first step to develop closed form expressions of the different sensitivities is to obtain a general expression of the network variables as a function of the machine internal nodes. To this aim it is possible to write nodal voltage equations that extended to the machine internal nodes, as shown in Fig. 8. To include $E_{j}^{\prime}$, the voltage behind transient reactaces $x_{d j}^{\prime}$, we add $N$ buses to the $n$-bus power system network, thus extending the admittance matrix to the machine internal nodes.

The internal machine buses are denoted by $n+1, \ldots, n+$ $j, \ldots, n+N$, which are the buses behind the transient reactances, $x_{d j}^{\prime}$. The resulting admittance matrix differs from the admittance matrix used in load flow analysis in that the additional internal machine buses are included to account for the machine internal voltages, $\tilde{E}_{j}^{\prime}$. In addition, loads are modeled as constant admittances and included in the diagonal elements of the admittances of the extended $\tilde{\boldsymbol{Y}}$ matrix. As a result, injected currents in all nodes other than the generator internal nodes are zero, i.e. $\tilde{I}_{i}=0$, $i=1, \ldots, n$.

Denoting all the generator current injections as $\tilde{\boldsymbol{I}}_{N}$, generator voltages as $\tilde{\boldsymbol{E}}_{N}^{\prime}$, and bus voltages as $\tilde{\boldsymbol{V}}_{n}$, the node voltage equations are

$$
\left[\begin{array}{c}
\mathbf{0} \\
\tilde{\boldsymbol{I}}_{N}
\end{array}\right]=\left[\begin{array}{cc}
\tilde{\boldsymbol{Y}}_{n n} & \tilde{\boldsymbol{Y}}_{n N} \\
\tilde{\boldsymbol{Y}}_{n N}^{T} & \tilde{\boldsymbol{Y}}_{N N}
\end{array}\right]\left[\begin{array}{c}
\tilde{\boldsymbol{V}}_{n} \\
\tilde{\boldsymbol{E}}_{N}^{\prime}
\end{array}\right]
$$

From (12) an expression for the bus voltage phasors as a function of the machine internal voltages is obtained as

$$
\tilde{\boldsymbol{V}}_{n}=-\tilde{\boldsymbol{Y}}_{n n}^{-1} \tilde{\boldsymbol{Y}}_{n N} \tilde{\boldsymbol{E}}_{N}^{\prime}
$$

where $\tilde{\boldsymbol{Y}}_{n n}^{-1} \tilde{\boldsymbol{Y}}_{n N}$ is referred to as the bus voltage reconstruction matrix and has a size of $(n \times N)$. Let the bus voltage reconstruction coefficient matrix be given by

$$
\tilde{\boldsymbol{\kappa}}=-\tilde{\boldsymbol{Y}}_{n n}^{-1} \tilde{\boldsymbol{Y}}_{n N}=\boldsymbol{\kappa} \angle \boldsymbol{\gamma}
$$




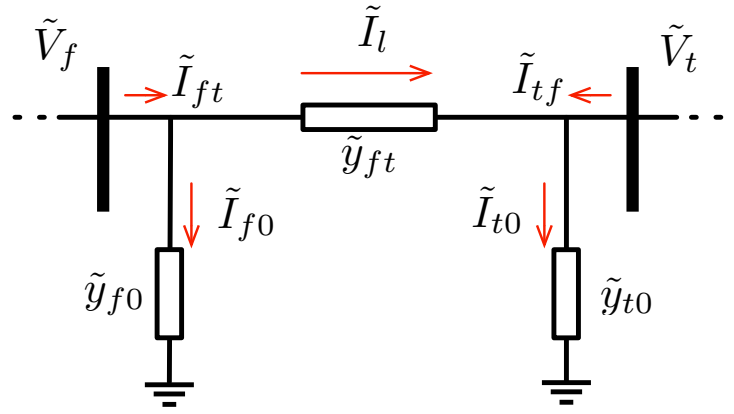

Figure 9: Current flows in the $\pi$-model of a transmission line

where $\boldsymbol{\kappa}$ and $\boldsymbol{\gamma}$ are the magnitude and angle of $\tilde{\boldsymbol{k}}$. We can now obtain a generalized expression relating the voltage phasor at bus $i$ with the machine internal voltages using (14)

$$
\tilde{V}_{i}=\tilde{\kappa}_{i 1} \tilde{E}_{1}^{\prime}+\tilde{\kappa}_{i 2} \tilde{E}_{2}^{\prime}+\ldots+\tilde{\kappa}_{i N} \tilde{E}_{N}^{\prime}=\sum_{j=1}^{N} \tilde{\kappa}_{i j} \tilde{E}_{j}^{\prime}
$$

Hence the voltage at the $i$-th bus is a function of the machine internal angles $\delta_{j}$. In this expression, $\tilde{V}_{i}$ depends on the value of the machine internal voltage magnitudes at the equilibrium, $E_{j}^{\prime}$, and the admittances in the voltage reconstruction coefficient matrix $\tilde{\kappa}_{i j}$, with $j=1, \ldots, N$.

It is also possible to develop a generalized expression for the complex line current flow through any line of the power network. Consider the $\pi$-equivalent of a transmission line shown in Fig. 9, the current from bus $f$ to bus $t$ is given by

$$
\tilde{I}_{f t}=\left(\tilde{y}_{f t}+\tilde{y}_{f 0}\right) \tilde{V}_{f}-\tilde{y}_{f t} \tilde{V}_{t}
$$

Letting $\tilde{Y}_{f t 0}=\tilde{y}_{f t}+\tilde{y}_{f 0}$ and $\tilde{Y}_{f t}=\tilde{y}_{f t}$, and writing the voltage for buses $f$ and $j$ in terms of the internal machine voltages using (15), we obtain

$$
\tilde{I}_{f t}=\sum_{j=1}^{N}\left(\tilde{Y}_{f t 0} \tilde{\kappa}_{f j}-\tilde{Y}_{f t} \tilde{\kappa}_{t j}\right) \tilde{E}_{j}^{\prime}=\sum_{j=1}^{N} \tilde{\Psi}_{F T j} \tilde{E}_{j}^{\prime}
$$

where $\tilde{\Psi}_{F T j}=\Psi_{F T j} \angle \psi_{F T j}$, and

$$
\begin{aligned}
& \Psi_{F T j}=\left|\tilde{Y}_{f t 0} \tilde{\kappa}_{f j}-\tilde{Y}_{f t} \tilde{\kappa}_{t j}\right|, \\
& \psi_{F T j}=\angle\left(\tilde{Y}_{f t 0} \tilde{\kappa}_{f j}-\tilde{Y}_{f t} \tilde{\kappa}_{t j}\right)
\end{aligned}
$$

The complex line current flow in Line $f$ - $t$ is a function of the machine angles $\delta_{j}$. The phasor $\tilde{I}_{f t}$ depends on the value of the machine internal voltage magnitudes at the equilibrium, $E_{j}^{\prime}$, the admittances in the voltage reconstruction coefficient matrix $\tilde{\boldsymbol{\kappa}}_{\boldsymbol{i} \boldsymbol{j}}$, and the admittances and shunts between buses $f$ and $t$. Expressions (15) and (17) are used to derive closed form expressions of the network sensitivities, a complete derivation can be found in [12].
4.2.1 Voltage Sensitivities. Given an $N$-machine power system, the bus voltage magnitude sensitivities for the $i$-th bus with respect to the $j$-th machine angle are given by

$$
\frac{\partial V_{i}}{\partial \delta_{j}}=\frac{1}{\left|\tilde{V}_{i}\right|}(\alpha), j=p ; \frac{\partial V_{i}}{\partial \delta_{j}}=\frac{1}{\left|\tilde{V}_{i}\right|}(-\alpha), j \neq p
$$

where

$$
\begin{gathered}
\left|\tilde{V}_{i}\right|=\sqrt{\left(\sum_{j=1}^{N} \tilde{\kappa}_{i j} \tilde{E}_{j}^{\prime}\right)^{2}} \\
\alpha=-\sum_{p=1}^{N-1} \sum_{\substack{q=p+1 \\
N=1}}^{N} \kappa_{i p} \kappa_{i q} E_{p}^{\prime} E_{q}^{\prime} \sin \left(\delta_{p}+\gamma_{i p}-\delta_{q}-\gamma_{i q}\right) \\
+\sum_{\substack{p=1 \\
p \neq j}}^{N-1} \sum_{\substack{q=p+1 \\
q \neq j}}^{N=p+1} \kappa_{i p} \kappa_{i q} E_{p}^{\prime} E_{q}^{\prime} \sin \left(\delta_{p}+\gamma_{i p}-\delta_{q}-\gamma_{i q}\right)
\end{gathered}
$$

For the bus voltage angle

$$
\begin{aligned}
\frac{\partial \theta_{i}}{\partial \delta_{j}}= & \frac{1}{\left|\tilde{V}_{i}\right|^{2}}\left[\kappa_{i j}^{2} E_{j}^{\prime 2}+\right. \\
& \left.\sum_{\substack{q=1 \\
q \neq j}}^{N} \kappa_{i j} \kappa_{i q} E_{j}^{\prime} E_{q}^{\prime} \cos \left(\delta_{j}+\gamma_{i j}-\delta_{q}-\gamma_{i q}\right)\right]
\end{aligned}
$$

is the closed form formula to obtain the sensitivity of the $i$-th bus voltage angle to the $j$-th machine angle.

4.2.2 Current Sensitivities. The line current magnitude sensitivities in any line from bus $f$ to bus $t$ with respect to the $j$-th machine angle are given by

$\frac{\partial I_{f t}}{\partial \delta_{j}}=\frac{1}{\left|\tilde{I}_{f t}\right|}(\beta), j=p ; \frac{\partial I_{f t}}{\partial \delta_{j}}=\frac{1}{\left|\tilde{I}_{f t}\right|}(-\beta), j \neq p$

where

$$
\left|\tilde{I}_{f t}\right|=\sqrt{\left(\sum_{j=1}^{N} \tilde{\Psi}_{F T j} \tilde{E}_{j}^{\prime}\right)^{2}}
$$

is the equilibrium value of the line current magnitude, and $\beta$ is given by (25) at the top of p. 7 .

For the line current angle

$$
\begin{aligned}
& \frac{\partial \phi_{f t}}{\partial \delta_{j}}=\frac{1}{\left|\tilde{I}_{f t}\right|^{2}}\left[\Psi_{F T j}^{2} E_{j}^{\prime 2}+\right. \\
& \left.\quad \sum_{\substack{q=1 \\
q \neq j}}^{N} \Psi_{F T j} \Psi_{F T q} E_{j}^{\prime} E_{q}^{\prime} \cos \left(\delta_{j}+\psi_{F T j}-\delta_{q}-\psi_{F T q}\right)\right]
\end{aligned}
$$

is the closed form formula to obtain the sensitivity of $\phi_{f t}$ with respect to the $j$-th machine angle.

Finally, the sensitivities of the real and imaginary part of the line current (16) are given by

$$
\begin{aligned}
& \frac{\partial \Re\left\{\tilde{I}_{f t}\right\}}{\partial \delta_{j}}=-\sum_{j=1}^{N} \Psi_{F T j} E_{j}^{\prime} \sin \left(\delta_{j}+\psi_{F T j}\right) \\
& \frac{\partial \Im\left\{\tilde{I}_{f t}\right\}}{\partial \delta_{j}}=\sum_{j=1}^{N} \Psi_{F T j} E_{j}^{\prime} \cos \left(\delta_{j}+\psi_{F T j}\right)
\end{aligned}
$$

Similar expressions can be obtained for the current from bus $t$ to $f$ by substituting the subscripts $f t$ and $F T$ with $t f$ and $T F$, and using the appropriate coefficients and admittances. 


$$
\begin{aligned}
\beta=-\sum_{p=1}^{N-1} \sum_{q=p+1}^{N} \Psi_{F T p} \Psi_{F T q} E_{p}^{\prime} E_{q}^{\prime} \sin \left(\delta_{p}+\psi_{F T p}-\delta_{q}-\psi_{F T q}\right) \\
\quad+\sum_{\substack{p=1 \\
p \neq j}}^{N-1} \sum_{\substack{q=p+1 \\
q \neq j}}^{N} \Psi_{F T p} \Psi_{F T q} E_{p}^{\prime} E_{q}^{\prime} \sin \left(\delta_{p}+\psi_{F T p}-\delta_{q}-\psi_{F T q}\right)
\end{aligned}
$$

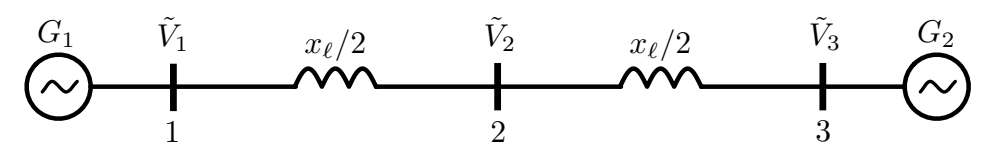

(a) Two-machine 3-bus system

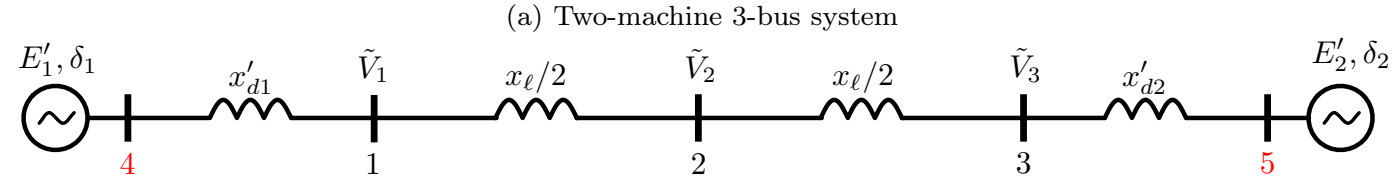

(b) Two-machine system extended to the machine internal nodes

Figure 10: Two-machine system one-line diagram and its extension to the machine internal nodes

\subsection{Explicit Sensitivity Expressions for a Longitudinal Two-Machine System}

As an illustration, explicit sensitivity expressions for the two-machine system with the parameters given in Fig. 10a are derived. Figure 10b shows the network extended to the machine internal nodes. The admittance matrix extended to the machine internal nodes is

$$
\begin{aligned}
\tilde{\boldsymbol{Y}} & =\left[\begin{array}{ccc|cc}
\tilde{Y}_{11} & \tilde{Y}_{12} & 0 & \tilde{Y}_{14} & 0 \\
\tilde{Y}_{21} & \tilde{Y}_{22} & \tilde{Y}_{23} & 0 & 0 \\
0 & \tilde{Y}_{32} & \tilde{Y}_{33} & 0 & \tilde{Y}_{35} \\
\hline \tilde{Y}_{41} & 0 & 0 & \tilde{Y}_{44} & 0 \\
0 & 0 & \tilde{Y}_{53} & 0 & \tilde{Y}_{55}
\end{array}\right] \\
& =\left[\begin{array}{ccc|cc}
-j \frac{x_{\ell}+2 x_{d 1}}{x_{d 1} x_{\ell}} & j \frac{2}{x_{\ell}} & 0 & j \frac{1}{x_{d 1}^{\prime}} & 0 \\
j \frac{2}{x_{\ell}} & -j \frac{4}{x_{\ell}} & j \frac{2}{x_{\ell}} & 0 & 0 \\
0 & j \frac{2}{x_{\ell}} & -j \frac{x_{\ell}+2 x_{d 2}}{x_{d 2} x_{\ell}} & 0 & j \frac{1}{x_{d 2}^{\prime}} \\
\hline j \frac{1}{x_{d 1}^{\prime}} & 0 & 0 & -j \frac{1}{x_{d 1}^{\prime}} & 0 \\
0 & 0 & j \frac{1}{x_{d 2}^{\prime}} & 0 & -j \frac{1}{x_{d 2}^{\prime}}
\end{array}\right]
\end{aligned}
$$

from which the bus voltage reconstruction coefficients can be computed using (14). Substituting the parameters shown in Fig. 10b, and letting

$$
\begin{aligned}
\operatorname{det}\left(\tilde{\boldsymbol{Y}}_{n n}\right) & =\tilde{Y}_{11} \tilde{Y}_{22} \tilde{Y}_{33}-\tilde{Y}_{11} \tilde{Y}_{23}^{2}-\tilde{Y}_{33} \tilde{Y}_{12}^{2} \\
& =j 4\left(\frac{x_{d 1}^{\prime}+x_{d 2}^{\prime}+x_{\ell}}{x_{d 1}^{\prime} x_{d 2}^{\prime} x_{\ell}^{2}}\right)
\end{aligned}
$$

we obtain the $\tilde{\boldsymbol{\kappa}}$ matrix

$$
\tilde{\boldsymbol{\kappa}}=\left[\begin{array}{cc}
\frac{x_{\ell}+x_{d 2}^{\prime}}{x_{d 1}^{\prime}+x_{d 2}^{\prime}+x_{\ell}} & \frac{x_{d 1}^{\prime}}{x_{d 1}^{\prime}+x_{d 2}^{\prime}+x_{\ell}} \\
\frac{1}{2}\left(\frac{2 x_{d 2}^{\prime}+x_{\ell}}{x_{d 1}^{\prime}+x_{d 2}^{\prime}+x_{\ell}}\right) & \frac{1}{2}\left(\frac{2 x_{d 1}+x_{\ell}}{x_{d 1}^{\prime}+x_{d 2}^{\prime}+x_{\ell}}\right) \\
\frac{x_{d 2}^{\prime}}{x_{d 1}^{\prime}+x_{d 2}^{\prime}+x_{\ell}} & \frac{x_{\ell}+x_{d 1}^{\prime}}{x_{d 1}^{\prime}+x_{d 2}^{\prime}+x_{\ell}}
\end{array}\right]
$$

note that the phase angles of the elements of $\tilde{\boldsymbol{\kappa}}$ are zero, i.e., $\gamma=0$. The bus voltage magnitude sensitivities can be obtained as follows. For Bus 1 they are

$$
\frac{\partial V_{1}}{\partial \delta_{1}}=-\frac{\operatorname{num}_{\partial V_{1} \partial \delta_{1}}}{\operatorname{den}_{\partial V_{1} \partial \delta_{1}}} \quad(31) \quad \frac{\partial V_{1}}{\partial \delta_{2}}=-\frac{\partial V_{1}}{\partial \delta_{1}}
$$
where

$$
\begin{aligned}
& \operatorname{num}_{\partial V_{1} \partial \delta_{1}}=x_{d 1}^{\prime}\left(\frac{x_{\ell}+x_{d 2}^{\prime}}{x_{d 1}^{\prime}+x_{d 2}^{\prime}+x_{\ell}}\right) E_{1}^{\prime} E_{2}^{\prime} \sin \left(\delta_{1}-\delta_{2}\right) \\
& \operatorname{den}_{\partial V_{1} \partial \delta_{1}}=\left[\left(x_{\ell}+x_{d 2}^{\prime}\right)^{2} E_{1}^{\prime 2}+x_{d 1}^{\prime 2} E_{2}^{\prime 2}\right. \\
& \left.+2 x_{d 1}^{\prime}\left(x_{\ell}+x_{d 2}^{\prime}\right) E_{1}^{\prime} E_{2}^{\prime} \cos \left(\delta_{1}-\delta_{2}\right)\right]^{\frac{1}{2}}
\end{aligned}
$$

For Bus 2 the voltage magnitude sensitivities are where

$$
\frac{\partial V_{2}}{\partial \delta_{1}}=-\frac{1}{2} \frac{\operatorname{num}_{\partial V_{2} \partial \delta_{1}}}{\operatorname{den}_{\partial V_{2}} \partial \delta_{1}} \quad(33) \quad \frac{\partial V_{2}}{\partial \delta_{2}}=-\frac{\partial V_{2}}{\partial \delta_{1}}
$$

$$
\begin{gathered}
\operatorname{num}_{\partial V_{2} \partial \delta_{1}}=\frac{\left(x_{\ell}+2 x_{d 1}^{\prime}\right)\left(x_{\ell}+2 x_{d 2}^{\prime}\right)}{x_{d 1}^{\prime}+x_{d 2}^{\prime}+x_{\ell}} E_{1}^{\prime} E_{2}^{\prime} \sin \left(\delta_{1}-\delta_{2}\right) \\
\operatorname{den}_{\partial V_{2} \partial \delta_{1}=} \begin{aligned}
{\left[\left(x_{\ell}+2 x_{d 2}^{\prime}\right)^{2} E_{1}^{\prime 2}+\left(x_{\ell}+2 x_{d 1}^{\prime}\right)^{2} E_{2}^{\prime 2}\right.} \\
\left.+2\left(x_{\ell}+x_{d 1}^{\prime}\right)\left(x_{\ell}+x_{d 2}^{\prime}\right) E_{1}^{\prime} E_{2}^{\prime} \cos \left(\delta_{1}-\delta_{2}\right)\right]^{\frac{1}{2}}
\end{aligned}
\end{gathered}
$$

and for Bus 3

$$
\frac{\partial V_{3}}{\partial \delta_{1}}=-\frac{\operatorname{num}_{\partial V_{3} \partial \delta_{1}}}{\operatorname{den}_{\partial V_{3} \partial \delta_{1}}} \quad(35) \quad \frac{\partial V_{3}}{\partial \delta_{2}}=-\frac{\partial V_{3}}{\partial \delta_{1}}
$$
where

$$
\begin{aligned}
\operatorname{num}_{\partial V_{3} \partial \delta_{1}}= & x_{d 2}^{\prime}\left(\frac{x_{\ell}+x_{d 1}^{\prime}}{x_{d 1}^{\prime}+x_{d 2}^{\prime}+x_{\ell}}\right) E_{1}^{\prime} E_{2}^{\prime} \sin \left(\delta_{1}-\delta_{2}\right) \\
\operatorname{den}_{\partial V_{3} \partial \delta_{1}=}= & {\left[x_{d 2}^{\prime 2} E_{1}^{\prime 2}+\left(x_{\ell}+x_{d 1}^{\prime}\right)^{2} E_{2}^{\prime 2}\right.} \\
& \left.+2 x_{d 2}^{\prime}\left(x_{\ell}+x_{d 1}^{\prime}\right) E_{1}^{\prime} E_{2}^{\prime} \cos \left(\delta_{1}-\delta_{2}\right)\right]^{\frac{1}{2}}
\end{aligned}
$$

The bus voltage angle sensitivities are, for Bus 1

$\frac{\partial \theta_{1}}{\partial \delta_{1}}=\frac{\operatorname{num}_{\partial \theta_{1} \partial \delta_{1}}}{\operatorname{den}_{\partial \theta_{1} \partial \delta_{1}}} \quad(37) \quad \frac{\partial \theta_{1}}{\partial \delta_{2}}=\frac{\operatorname{num}_{\partial \theta_{1} \partial \delta_{2}}}{\operatorname{den}_{\partial \theta_{1} \partial \delta_{2}}}$ where

$$
\operatorname{num}_{\partial \theta_{1} \partial \delta_{1}}=\left(x_{\ell}+x_{d 2}^{\prime}\right)^{2} E_{1}^{\prime 2}+x_{d 1}^{\prime}\left(x_{\ell}+x_{d 2}^{\prime}\right) E_{1}^{\prime} E_{2}^{\prime} \cos \left(\delta_{1}-\delta_{2}\right)
$$




$$
\begin{aligned}
\operatorname{den}_{\partial \theta_{1} \partial \delta_{1}=} & \left(x_{\ell}+x_{d 2}^{\prime}\right)^{2} E_{1}^{\prime 2}+x_{d 1}^{\prime 2} E_{2}^{\prime 2}+ \\
& 2 x_{d 1}^{\prime}\left(x_{\ell}+x_{d 2}^{\prime}\right) E_{1}^{\prime} E_{2}^{\prime} \cos \left(\delta_{1}-\delta_{2}\right) \\
\operatorname{num}_{\partial \theta_{1} \partial \delta_{2}}= & x_{d 1}^{\prime 2} E_{2}^{\prime 2}+x_{d 1}^{\prime 2}\left(x_{\ell}+x_{d 2}^{\prime}\right) E_{1}^{\prime} E_{2}^{\prime} \cos \left(\delta_{1}-\delta_{2}\right) \\
\operatorname{den}_{\partial \theta_{1} \partial \delta_{2}=}= & \left(x_{\ell}+2 x_{d 2}^{\prime}\right)^{2} E_{1}^{\prime 2}+x_{d 1}^{\prime 2} E_{2}^{\prime 2}+ \\
& 2 x_{d 1}^{\prime}\left(x_{\ell}+x_{d 2}^{\prime}\right) E_{1}^{\prime} E_{2}^{\prime} \cos \left(\delta_{1}-\delta_{2}\right)
\end{aligned}
$$

For Bus 2 the sensitivities are

where

$$
\frac{\partial \theta_{2}}{\partial \delta_{1}}=\frac{\operatorname{num}_{\partial \theta_{2} \partial \delta_{1}}}{\operatorname{den}_{\partial \theta_{2} \partial \delta_{1}}} \quad(39) \quad \frac{\partial \theta_{2}}{\partial \delta_{2}}=\frac{\operatorname{num}_{\partial \theta_{2} \partial \delta_{2}}}{\operatorname{den}_{\partial \theta_{2} \partial \delta_{2}}}
$$

$$
\begin{aligned}
\operatorname{num}_{\partial \theta_{2} \partial \delta_{1}}= & \left(x_{\ell}+2 x_{d 2}^{\prime}\right)^{2} E_{1}^{\prime 2}+ \\
& \left(x_{\ell}+2 x_{d 1}^{\prime}\right)\left(x_{\ell}+2 x_{d 2}^{\prime}\right) E_{1}^{\prime} E_{2}^{\prime} \cos \left(\delta_{1}-\delta_{2}\right) \\
\operatorname{den}_{\partial \theta_{2} \partial \delta_{1}}= & \left(x_{\ell}+2 x_{d 2}^{\prime}\right)^{2} E_{1}^{\prime 2}+\left(x_{\ell}+2 x_{d 1}^{\prime}\right) E_{2}^{\prime 2}+ \\
& 2\left(x_{\ell}+2 x_{d 1}^{\prime}\right)\left(x_{\ell}+2 x_{d 2}^{\prime}\right) E_{1}^{\prime} E_{2}^{\prime} \cos \left(\delta_{1}-\delta_{2}\right) \\
\operatorname{num}_{\partial \theta_{2} \partial \delta_{2}=}\left(x_{\ell}+2 x_{d 1}^{\prime}\right)^{2} E_{2}^{\prime 2}+ & \left(x_{\ell}+2 x_{d 1}^{\prime}\right)\left(x_{\ell}+2 x_{d 2}^{\prime}\right) E_{1}^{\prime} E_{2}^{\prime} \cos \left(\delta_{1}-\delta_{2}\right) \\
\operatorname{den}_{\partial \theta_{2} \partial \delta_{2}=}\left(x_{\ell}+2 x_{d 2}^{\prime}\right)^{2} E_{1}^{\prime 2}+\left(x_{\ell}+2 x_{d 1}^{\prime}\right) E_{2}^{\prime 2}+ & 2\left(x_{\ell}+2 x_{d 1}^{\prime}\right)\left(x_{\ell}+2 x_{d 2}^{\prime}\right) E_{1}^{\prime} E_{2}^{\prime} \cos \left(\delta_{1}-\delta_{2}\right)
\end{aligned}
$$

and for Bus 3 the sensitivities are

where

$$
\frac{\partial \theta_{3}}{\partial \delta_{1}}=\frac{\operatorname{num}_{\partial \theta_{3} \partial \delta_{1}}}{\operatorname{den}_{\partial \theta_{3} \partial \delta_{1}}} \quad \text { (41) } \quad \frac{\partial \theta_{3}}{\partial \delta_{2}}=\frac{\operatorname{num}_{\partial \theta_{3} \partial \delta_{2}}}{\operatorname{den}_{\partial \theta_{3} \partial \delta_{2}}}
$$

$$
\begin{aligned}
\operatorname{num}_{\partial \theta_{3} \partial \delta_{1}}= & x_{d 2}^{\prime 2} E_{1}^{\prime 2}+x_{d 2}^{\prime}\left(x_{\ell}+x_{d 1}^{\prime}\right) E_{1}^{\prime} E_{2}^{\prime} \cos \left(\delta_{1}-\delta_{2}\right) \\
\operatorname{den}_{\partial \theta_{3} \partial \delta_{1}}= & x_{d 2}^{\prime 2} E_{1}^{\prime 2}+\left(x_{\ell}+x_{d 1}^{\prime}\right)^{2} E_{2}^{\prime 2}+ \\
& 2 x_{d 2}^{\prime}\left(x_{\ell}+x_{d 1}^{\prime}\right) E_{1}^{\prime} E_{2}^{\prime} \cos \left(\delta_{1}-\delta_{2}\right) \\
\operatorname{num}_{\partial \theta_{3} \partial \delta_{2}}= & \left(x_{\ell}+x_{d 1}^{\prime}\right)^{2} E_{2}^{\prime 2}+x_{d 2}^{\prime}\left(x_{\ell}+x_{d 1}^{\prime}\right) E_{1}^{\prime} E_{2}^{\prime} \cos \left(\delta_{1}-\delta_{2}\right) \\
\operatorname{den}_{\partial \theta_{3} \partial \delta_{2}}= & x_{d 2}^{\prime 2} E_{1}^{\prime 2}+\left(x_{\ell}+x_{d 1}^{\prime}\right)^{2} E_{2}^{\prime 2}+ \\
& 2 x_{d 2}^{\prime}\left(x_{\ell}+x_{d 1}^{\prime}\right) E_{1}^{\prime} E_{2}^{\prime} \cos \left(\delta_{1}-\delta_{2}\right)
\end{aligned}
$$

More general expressions can be obtained for the twomachine system when $n$ buses are between the two machines. In such case, the generalized closed form expressions for the $i$-th bus of the two-machine power system will be given by

$$
\begin{aligned}
& \frac{\partial V_{i}}{\partial \delta_{1}}=-\frac{\operatorname{num}_{\partial V_{i} \partial \delta_{1}}}{\operatorname{den}_{\partial V_{i} \partial \delta_{1}}}(43) \quad \frac{\partial V_{i}}{\partial \delta_{2}}=-\frac{\partial V_{i}}{\partial \delta_{1}} \\
& \operatorname{num}_{\partial V_{i} \partial \delta_{1}}=\kappa_{i 1} \kappa_{i 2} E_{1}^{\prime} E_{2}^{\prime} \sin \left(\delta_{1}+\gamma_{i 1}-\delta_{2}-\gamma_{i 2}\right) \\
& \operatorname{den}_{\partial V_{i} \partial \delta_{1}}=\left[\kappa_{i 1}^{2} E_{1}^{\prime 2}+\kappa_{i 2}^{2} E_{2}^{\prime 2}+\right. \\
& \left.2 \kappa_{i 1} \kappa_{i 2} E_{1}^{\prime} E_{2}^{\prime} \cos \left(\delta_{1}+\gamma_{i 1}-\delta_{2}-\gamma_{i 2}\right)\right]^{\frac{1}{2}} \\
& \frac{\partial \theta_{i}}{\partial \delta_{1}}=\frac{\operatorname{num}_{\partial \theta_{i} \partial \delta_{1}}}{\operatorname{den}_{\partial \theta_{i} \partial \delta_{1}}} \quad(45) \quad \frac{\partial \theta_{i}}{\partial \delta_{2}}=\frac{\operatorname{num}_{\partial \theta_{i} \partial \delta_{2}}}{\operatorname{den}_{\partial \theta_{i} \partial \delta_{2}}}
\end{aligned}
$$

\subsection{Properties of the Network Sensitivities}

We complete this discussion by providing some intrinsic properties of the sensitivities discussed above. For the bus voltage sensitivities the properties are ${ }^{1}$

$$
\sum_{j=1}^{N}\left(\frac{\partial V_{i}}{\partial \delta_{j}}\right)=0, \quad \sum_{j=1}^{N}\left(\frac{\partial \theta_{i}}{\partial \delta_{j}}\right)=1
$$

and for the line currents the sensitivity properties are

$$
\sum_{j=1}^{N}\left(\frac{\partial I_{f t}}{\partial \delta_{j}}\right)=0, \quad \sum_{j=1}^{N}\left(\frac{\partial \phi_{f t}}{\partial \delta_{j}}\right)=1
$$

Both voltage and current sensitivities have the same property: the sum of sensitivities of the magnitude of the phasor

\footnotetext{
${ }^{1} \mathrm{~A}$ formal proof of these properties is found in the Appendix.
} 


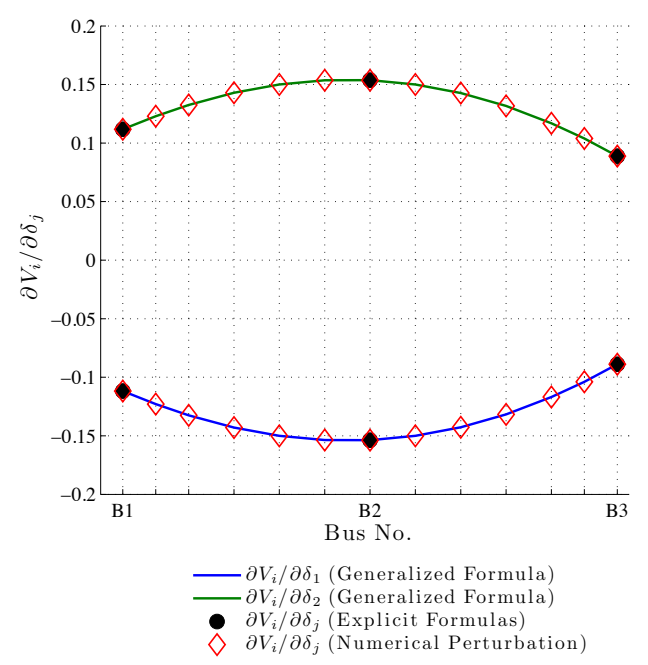

(a) Bus Voltage Magnitude Sensitivities

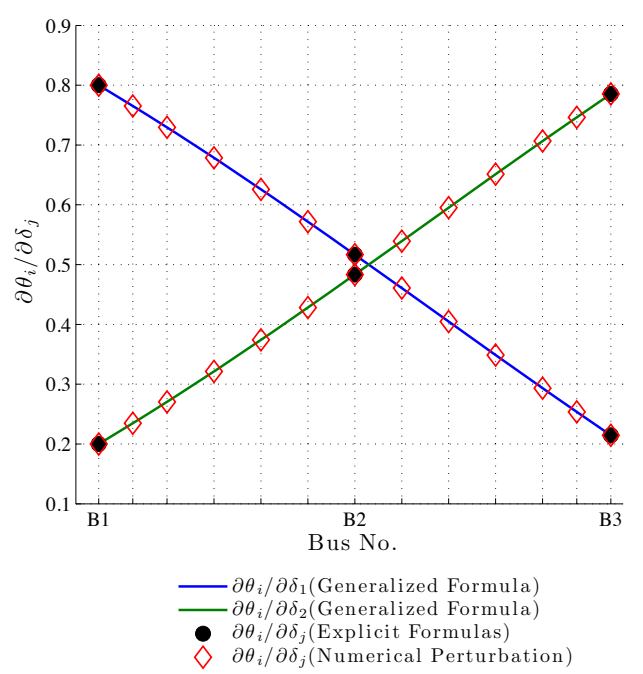

(b) Bus Voltage Angle Sensitivities

Figure 11: Comparison of Bus Voltage Sensitivities obtained from Explicit Analytical Expressions and Numerical Perturbation

w.r.t. all the machine angles is zero, while the sum of the sensitivities of the angle of the phasor w.r.t. machine angles is equal to unity.

These sensitivity properties can be explained by using the phasor diagram in Fig. 12. The voltage at the $i$-th bus of a two-machine system, $\tilde{V}_{i}=V_{i} \epsilon^{j \theta_{i}}$, is perturbed by introducing small changes to the machine angles, $\Delta \delta_{1}$ and $\Delta \delta_{2}$, resulting in the perturbed voltage $\tilde{V}_{i}^{*}=V_{i}^{*} \varepsilon^{j \theta_{i}^{*}}$. When the perturbations introduced to the machine angles are identical, the resulting perturbation to the bus angle will be equal to the value used to perturb the machines. The perturbation of each machine is appropriately scaled by the corresponding sensitivity. Hence, the sum of all sensitivities is one. Note that if only one of the machines is perturbed, the bus angle will only be changed in the proportion dictated by the sensitivity and the perturbation value.

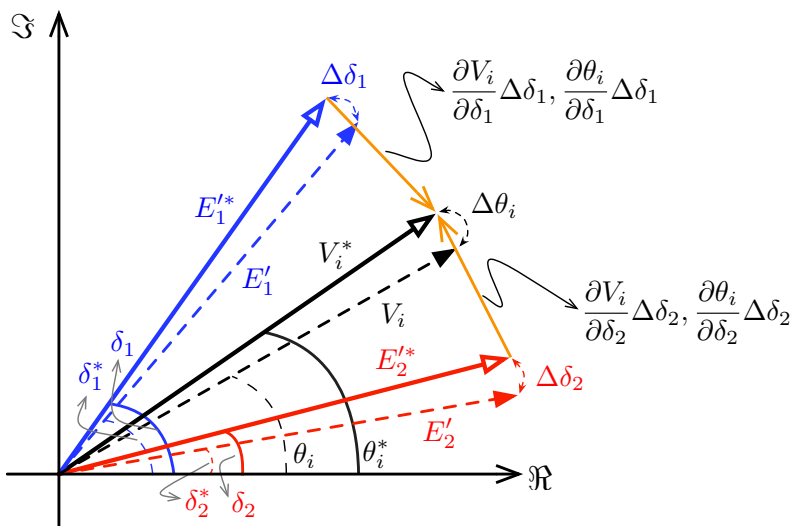

Figure 12: Illustration of the sensitivity properties

In addition, observe that the magnitude before the perturbation is the same as the magnitude after the perturbation, i.e. $V_{i}=V_{i}^{*}$. Note from the phasor diagram that $\Delta \delta_{1}$ scaled by $\partial V_{i} / \partial \delta_{1}$ will oppose the change in the voltage magnitude from $\Delta \delta_{2}$ scaled by $\partial V_{i} / \partial \delta_{2}$. Note that the value of the bus voltage sensitivities for this case is identical. Because both sensitivities are equal with opposing signs the change in the bus voltage magnitude, $\Delta V=0$. Hence, the sum of the sensitivities is zero.

\section{Sensitivity Mapping onto the Electrome- chanical Mode Shapes}

In this section we investigate how the eigenvector matrices discussed in Section 3, can be used to separate the components of each oscillatory mode contained in the total change of the network variables. This result was previously exploited in [8], where we showed that it is possible to compute the bus voltage magnitude and frequency mode shapes. Here we extend this concept to include any type of network variable, and to understand how by mapping the network sensitivities to a particular mode shape from $\bar{A}$ it is possible to replicate the phase shift observed in the modal components of PMU measurements.

By mapping the network sensitivities onto the right eigenvector we obtain a network modeshape which indicates the observability of a particular mode in a specific network variable. The mode shapes as observed in the bus voltage magnitudes and angles for all the network buses are given by

$$
\boldsymbol{S}_{\mathrm{V}}=\boldsymbol{C}_{\mathrm{V} \delta} \boldsymbol{W} \quad(49) \quad \boldsymbol{S}_{\theta}=\boldsymbol{C}_{\theta \delta} \boldsymbol{W}
$$

where $\boldsymbol{W}$ is of size $(N \times N)$.

Similarly, for the line current magnitude and angle we have

$$
\boldsymbol{S}_{\mathrm{I}_{\mathrm{ft}}}=\boldsymbol{C}_{\mathrm{I}_{\mathrm{ft}} \delta} \boldsymbol{W} \quad(51) \quad \boldsymbol{S}_{\phi_{\mathrm{ft}}}=\boldsymbol{C}_{\phi_{\mathrm{ft}} \delta} \boldsymbol{W}
$$

where the subscript $f t$ indicates that these are the modal components from bus $f$ to bus $t$. Modal components for any other network variable can be obtained in similar fashion. 
When the eigenvectors are computed from $\overline{\boldsymbol{A}}$, the phase shifts due to damping will be mapped onto the bus voltages in a proportion dictated by the sensitivities, therefore reproducing the phase shifts observed in the network variables measured by PMUs.

To illustrate, consider the two-area four-generator system discussed in Section 3.2. The bus voltage magnitude sensitivities of Bus 7 w.r.t. all machine angles are given by

$$
\boldsymbol{C}_{\mathrm{V} \delta(7, k)}=\left[\begin{array}{llll}
-0.0463 & -0.0270 & 0.0146 & 0.0587
\end{array}\right]
$$

Multiplying by the eigenvector column corresponding to the inter-area mode, the inter-area component of the bus voltage magnitude at Bus 7 is

$$
\begin{aligned}
& S_{\mathrm{V}_{(7,2)}=}=\frac{\partial V_{7}}{\partial \delta_{1}} W_{(1,2)}+\frac{\partial V_{7}}{\partial \delta_{2}} W_{(2,2)}+\frac{\partial V_{7}}{\partial \delta_{3}} W_{(3,2)}+\frac{\partial V_{7}}{\partial \delta_{4}} W_{(4,2)} \\
& S_{\mathrm{V}_{(7,2)}=}-0.0463 W_{(1,2)}-0.0270 W_{(2,2)}+0.0146 W_{(3,2)}+ \\
& 0.0587 W_{(4,2)}
\end{aligned}
$$

Observe how each sensitivity scales its corresponding element of inter-area mode shape.

The eigenvector matrix computed from (3) has the interarea mode shape given by Column 2 of (4)

$$
W_{(j, 2)}(\boldsymbol{A})=\left[\begin{array}{llll}
-0.5930 & -0.5997 & -0.4539 & 0.2876
\end{array}\right]^{T}
$$

Using this mode shape it is possible to compute the interarea component of the bus voltage magnitude at Bus 7 (54)

$$
\begin{array}{r}
S_{\mathrm{V}_{(7,2)}}(\boldsymbol{A})=(-0.0463)(-0.5930)+(-0.0270)(-0.5997)+ \\
(0.0146)(-0.4539)+(0.0587)(0.2876)=0.05391
\end{array}
$$

Similarly, if the eigenvector matrix is computed from model (1), the inter-area mode shape is given by Column 2 of (6), and the inter-area component of the bus voltage magnitude becomes

$$
S_{\mathrm{V}_{(7,2)}}(\overline{\boldsymbol{A}})=0.0534 \angle-5.25^{\circ}
$$

Comparing (57) to (56) helps in understanding the origin of the phase shifts observed from phasor measurement data, that is, the presence of damping.

\subsection{Illustration with the Two-Area Four-Machine System}

We extend our discussion to consider the different network variables across the entire power system. The bus voltage magnitude mode shapes (the mapping of the sensitivities onto the electromechanical mode shapes) for all network buses are shown in the one-line diagrams in Figs. 4 and 13 for the inter-area mode, for both the case without damping and with damping, respectively, and the phasor diagrams in Fig. 14. These figures clearly show that the damping in $\bar{A}$ will give rise to the phase shifts across all bus voltage magnitudes in the power system. Consider the phase shift introduced to the voltage magnitude at Bus 1: when no damping is included $S_{\mathrm{V}_{(1,2)}}(\boldsymbol{A})=0.0544 \angle 0^{\circ}$ and, with the effect of damping $S_{\mathrm{V}_{(1,2)}}(\overline{\boldsymbol{A}})=0.0547 \angle-7.14^{\circ}$, thus the shift of $7.14^{\circ}$ is due to damping. Similarly for Bus $4, S_{\mathrm{V}_{(4,2)}}(\boldsymbol{A})=$

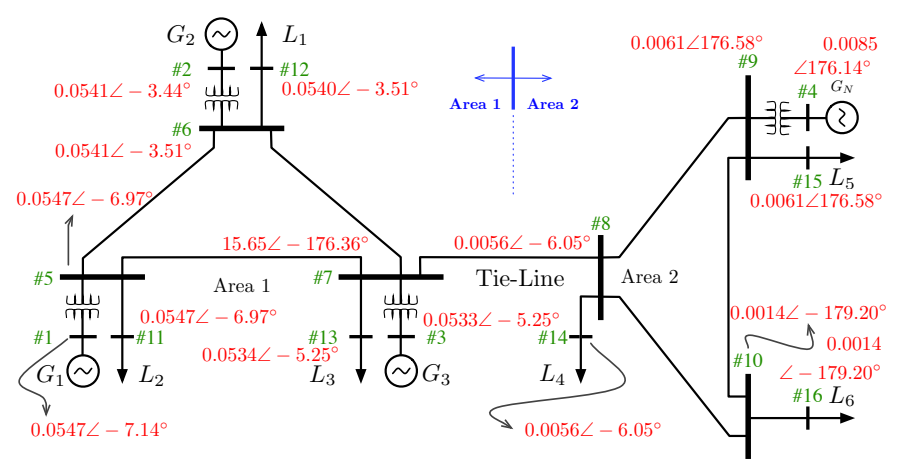

Figure 13: Two-Area Four-Machine Power System and Voltage Magnitude Oscillations from the Inter-area Mode (Model with Damping)

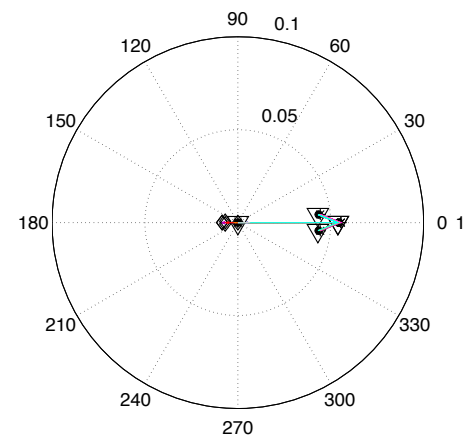

(a) Without Damping

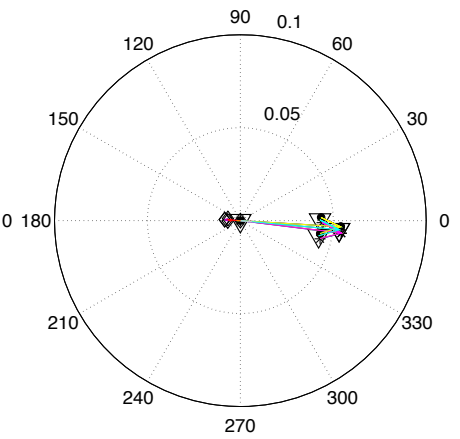

(b) With Damping

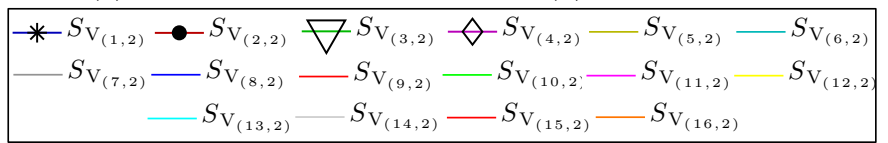

Figure 14: $V_{i}$ Inter-area mode shape with and without damping

$0.0085 \angle 180^{\circ}$, while $S_{\mathrm{V}_{(4,2)}}(\overline{\boldsymbol{A}})=0.0085 \angle 176.14^{\circ}$, the $3.86^{\circ}$ of difference between these two last quantities are a result of including damping. A more interesting case is Bus 8 which lies at the right end of the tie-line. Observe that $S_{\mathrm{V}_{(8,2)}}(\boldsymbol{A})=0.0057 \angle 0^{\circ}$, while $S_{\mathrm{V}_{(4,2)}}(\overline{\boldsymbol{A}})=0.0056 \angle-6.05^{\circ}$, the $-6.05^{\circ}$ being a result of the inclusion of damping.

It is worthwhile to note that these holds for any mode, for example, consider the bus voltage magnitude mode shape for Local Mode 1 shown in Fig. 15. In this case there is a more prominent phase shift between Buses 1 and 12 than in Fig. 14 because Local Mode 1 mostly involves $G_{1}$ and $G_{2}$, this is also reflected in the voltage mode shapes. It is also important to highlight that the observations above also hold for any network variable. Consider the bus voltage angle mode shapes in Fig. 16. In the case without damping in Fig. 16a it is observed that the bus voltage angles are either completely in phase or in anti-phase. In contrast, Fig. 16b shows that the result of including damping is to have phase shifts in all the mode shape. Another important feature of this mode shape is that when comparing Fig. 16b to Fig. $6 \mathrm{~b}$ it is realized that the machine angles outline a boundary within which the bus voltage angles exist. In 

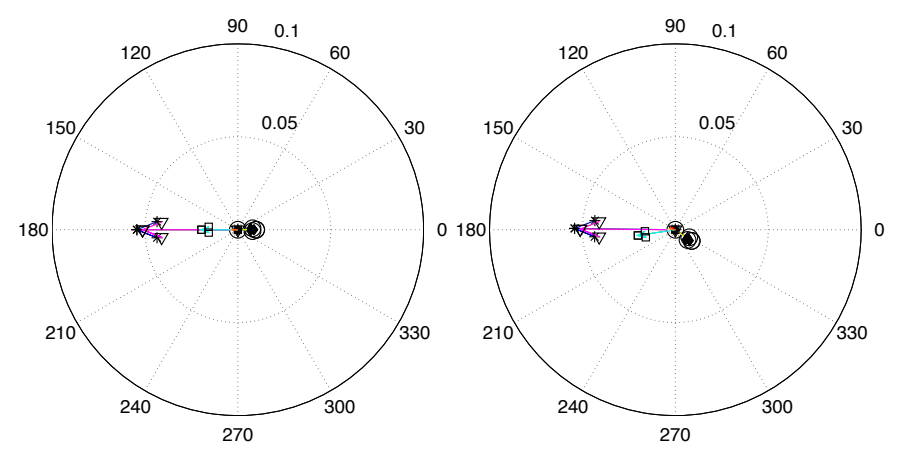

(a) Without Damping

(b) With Damping

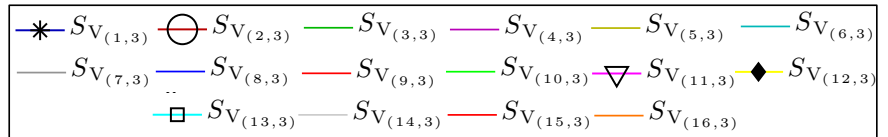

Figure 15: $V_{i}$ Local 1 mode shape with and without damping

other words, a bus voltage angle close to a generator will have a smaller relative phase angle with the generator than other further apart from it.

The relative time delay between the voltage magnitudes at Buses $p$ and $q$, can be calculated from the oscillatory frequency of the $k$-th mode and the phase shift at each bus by computing

$$
\tau_{(p-q, k)}=\frac{\theta_{(p, k)}-\theta_{(q, k)}}{2 \pi} \times \frac{1}{f_{k}}
$$

where $f_{k}$ is the frequency of the $k$-th mode of the system, and

$$
\theta_{(p, k)}=\angle\left(S_{\mathrm{V}_{(\mathrm{p}, \mathrm{k})}}\right), \theta_{(q, k)}=\angle\left(S_{\mathrm{V}_{(\mathrm{q}, \mathrm{k})}}\right)
$$

are the $k$-th mode phase shifts of the voltage magnitude oscillation in rad. at Buses $p$ and $q$, respectively, and $j=$ $1, \ldots, N$.

As an example consider the oscillations from Local Mode 1 at Bus $12, S_{\mathrm{V}_{(12,3)}}$, and Bus $1, S_{\mathrm{V}_{(1,3)}}$, as shown in Fig. 15. The relative time delay is given by

$$
\tau_{(12-1,3)}=\frac{(5.6295)-(3.1291)}{2 \pi \times 0.21516}=1.8495 \mathrm{sec}
$$

this time delay is shown in Fig. 17, where we compare oscillations for Local Mode 1 in the bus voltage magnitude at Bus 1 and Bus 12 .

Equation (58) can be used to compute the time delays for any network oscillation by selecting the network sensitivity and mode of interest. Conversely, the relative phase shift between the buses can also be computed by knowledge of the time-response of the network from

$$
\theta_{(p, k)}-\theta_{(q, k)}=2 \pi f_{k} \tau_{(p-q, k)}
$$

where $f_{k}$ is the frequency of the $k$-th mode of the system, note that this frequency is different for any system mode, i.e., the inter-area mode frequency is different from the Local Mode 1 frequency. Using the expression above and selecting a reference voltage magnitude we can obtain the

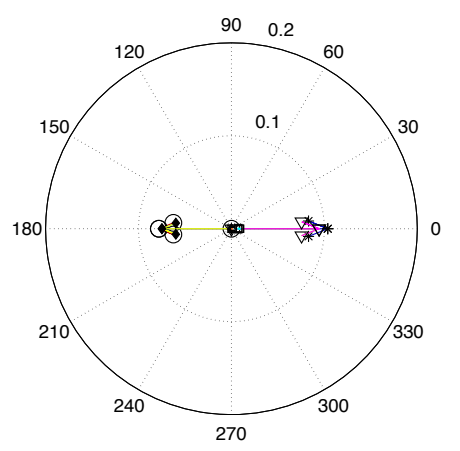

(a) Without Damping

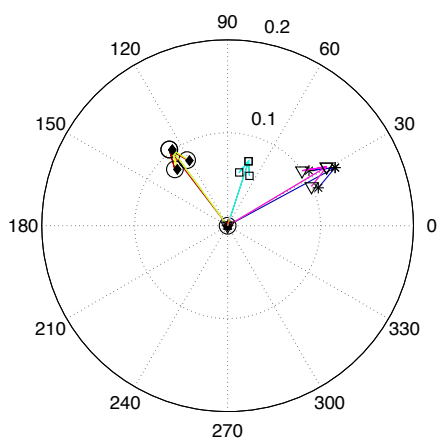

(b) With Damping
Figure 16: $\theta_{i}$ Local 1 mode shapes with and without damping

mode shapes from a time response, or more importantly, from PMU data.

Also of interest is the mapping for the complex line current flows. In Fig. 18 the mapping of $\boldsymbol{W}(\overline{\boldsymbol{A}})$ for the complex line current flow is shown. Note from Fig. 18 that the phase shift is also mapped onto these network variables. Moreover, the phase lags shown in the phasor diagram in Fig. 18a will translate to the different time delays that can be observed in Fig. 18b. More important, it is interesting to observe how the oscillations distribute in different lines as shown in the one-line diagram in Fig. 18c. Note that in Fig. 18c, it is possible to compute the current balance for the real and imaginary part, and that we can also determine how the oscillations are distributed in each line. This new capability to "track" the oscillations has important implications as discussed next.

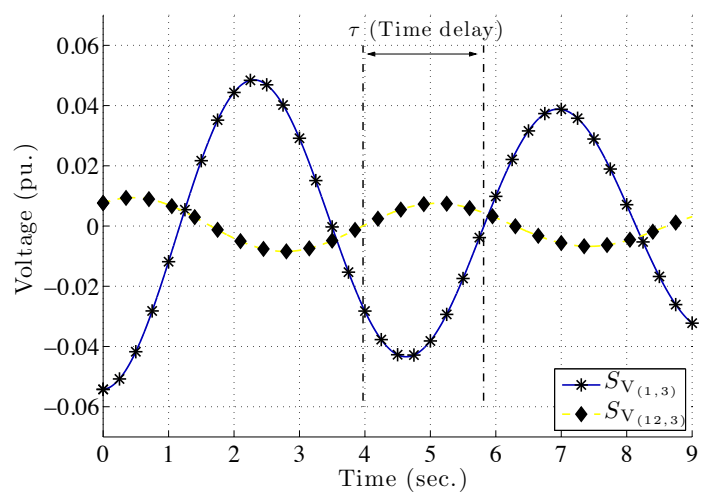

Figure 17: Time delay between $S_{\mathrm{V}_{(12,3)}}$ and $S_{\mathrm{V}_{(1,3)}}$

\section{Application for Inter-area Mode Tracing, PMU Siting, and Control Signal Synthe- sis}

This section outlines several applications network originating from this new understanding of power system oscillations. 


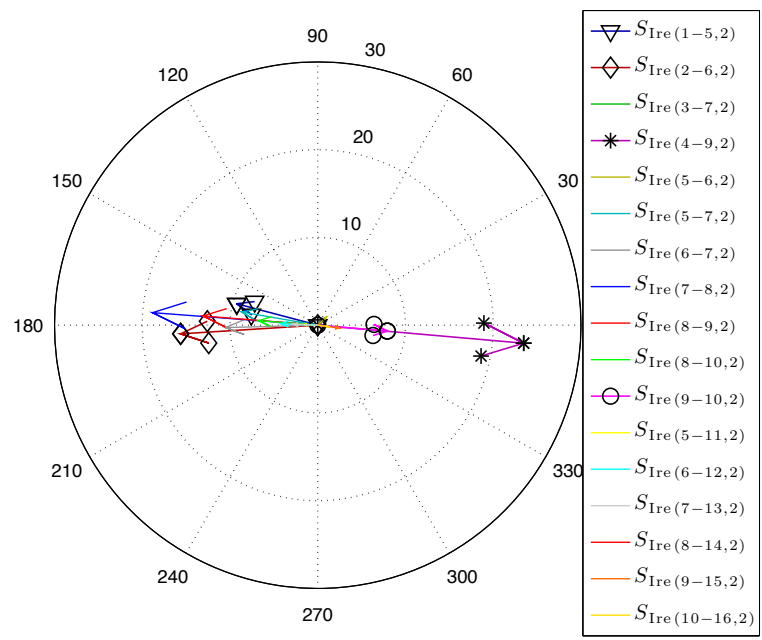

(a) Real Line Current Mode Shape - Damping

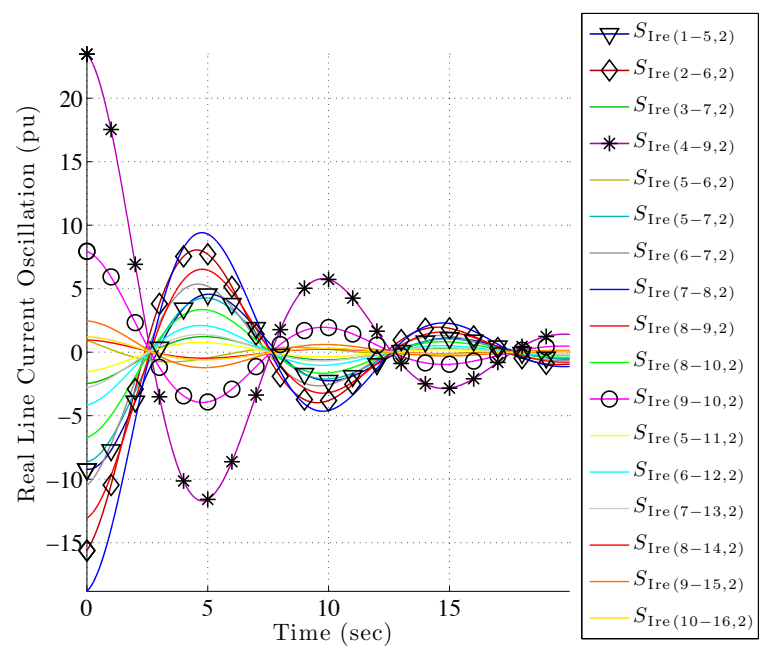

(b) Real Line Current Oscillations - Damping

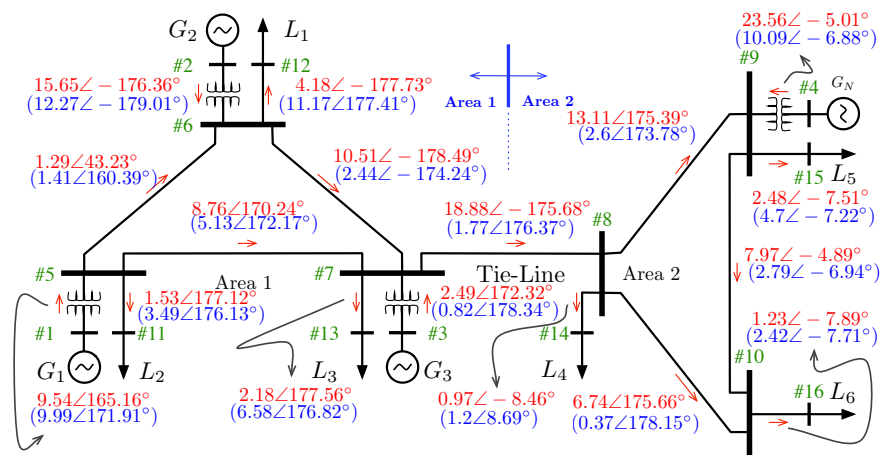

(c) Real and Imaginary Line Current Mode Oscillations in the Network - with Damping

Figure 18: Inter-area Mode $\tilde{I}_{f t}$ Mode Shapes with and without Damping (Imaginary quantities in the one-line diagrams are shown inside parenthesis)

The first use of the previous results is mode "tracing" or "tracking". Consider for example the line current flows in Fig. 18c. From Line 7-8, it is possible to see that the largest inter-area component in the line is provided by the current flow coming from Line 8-9. Tracing back this current flow, we see that the largest proportion of the inter-area

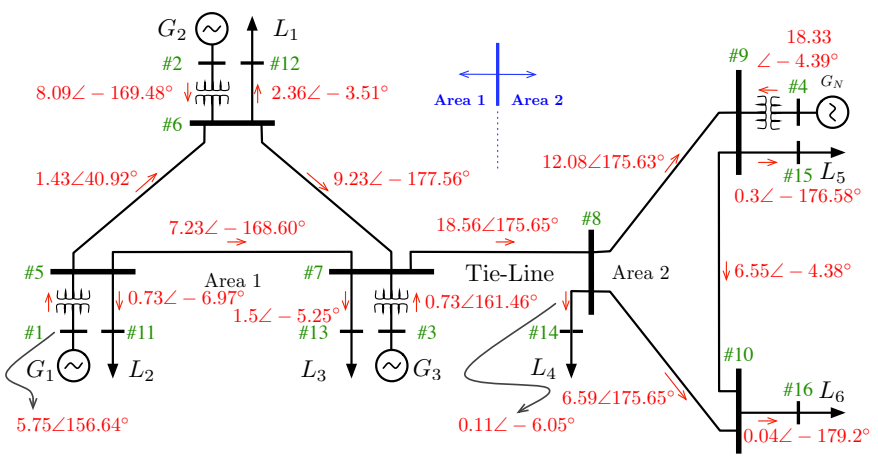

Figure 19: Inter-area Mode Active Power Mode Shapes

mode contributions of $G_{4}$ goes directly through Line 8-9, while the remaining contribution of $G_{4}$ first has to be distributed among Loads $L_{5}, L_{6}$, and $L_{14}$ before reaching Line 7-8. This new ability to trace the propagation of inter-area modes is certainly of interest when analyzing power system dynamics over a large network, and it has many additional implications, one being measurement placement. For example, if a local or remote current measurement is to be used as input signal for damping control in $G_{4}$, it becomes obvious from the discussion above that the line where the mode is the most observable is Line $8-9$, and thus, this line is the best candidate location to place the PMU for such an application. The mapping for the active power flows has similar characteristics and implications as depicted in the network diagram in Fig. 19 showing the inter-area components of the active power flow components. The placement will become more obvious as the transmission lines with the highest inter-area components should be prioritized to be equipped with PMUs.

In the network in Fig. 19 there is only one line interconnecting Area 1 and Area 2. For the sake of discussion, consider the case when multiple lines are connecting the areas as in [8]. By applying the mapping it is possible to determine which line will carry the highest contribution of the inter-area oscillation. The placement will also become more obvious as the transmission lines with the highest interarea components should be prioritized to be equipped with PMUs.

Line current magnitudes and remote bus voltage angles with relevant modal contents are in general desirable feedback control signals for FACTS controllers [18, 19]. Our analysis here will clearly show the magnitude of the interarea mode present in the signals. In the case of using remote bus voltage angles, this approach will be helpful in selecting two bus locations whose bus angle difference contains predominantly the inter-area mode to be controlled. The phase of the inter-area mode would be useful in designing the appropriate phase compensation in a damping controller.

Although not discussed in this paper, a signal having robust inter-area mode contents under various contingency conditions would be more preferable for feedback control. 


\section{Conclusions}

The results presented in this paper provide a novel understanding of power system oscillations as viewed from network variables. Furthermore, they have many potential applications for inter-area mode monitoring, PMU placement, and damping of power system oscillations.

\section{Acknowledgment}

This work was supported in part by the RPI Power System Research Consortium Industry Members: AEP, FirstEnergy, ISO NE, NYISO, and PJM, and by NSF through grant ECS-0622119.

We would like to thank Ritchie Carrol, Paul Trachian, and Lisa Beard of TVA; David Bertagnolli of ISO-NE, and Tony Weekes of Manitoba Hydro for providing PMU data from the Florida Disturbance event.

The help of Miss Katherine Elkington of KTH-EPS in providing a $\mathrm{LTT}_{\mathrm{EX}}$ template meeting the IREP guidelines is very appreciated.

\section{Notation}

\begin{tabular}{|c|c|}
\hline $\boldsymbol{x}$ & state vector \\
\hline $\boldsymbol{A}$ & system state matrix without damping \\
\hline $\bar{A}$ & system state matrix with damping \\
\hline $\boldsymbol{W}$ & right eigenvector matrix or mode shapes \\
\hline$i=1, \ldots, n$ & $\begin{array}{l}\text { buses in the network, } n \text { being the } \\
\text { total no. of buses }\end{array}$ \\
\hline$j=1, \ldots, N$ & $\begin{array}{l}\text { machines in the network, } N \text { being the } \\
\text { total no. of machines }\end{array}$ \\
\hline$\delta_{j}, \omega_{j}$ & $\begin{array}{l}\text { internal machine angle and } \\
\text { speed for the } j \text {-th machine }\end{array}$ \\
\hline$\tilde{V}_{i}, V_{i}, \theta_{i}$ & $\begin{array}{l}\text { bus voltage phasor, magnitude, } \\
\text { and angle for the } i \text {-th bus }\end{array}$ \\
\hline$f, t$ & "from" bus, "to" bus \\
\hline$\ell$ & total number of lines in the power system \\
\hline$\tilde{I}_{f t}, I_{f t}, \phi_{f t}$ & $\begin{array}{l}\text { line current phasor, magnitude, } \\
\text { and angle for line } f t\end{array}$ \\
\hline$\Delta \delta$ & vector of perturbed machine angles \\
\hline$\Delta V, \Delta \theta$ & $\begin{array}{l}\text { vector of perturbed bus voltage magnitudes } \\
\text { and angles }\end{array}$ \\
\hline $\boldsymbol{\Delta} \boldsymbol{I}_{\mathrm{ft}}, \boldsymbol{\Delta} \phi_{\mathrm{ft}}$ & $\begin{array}{l}\text { vector of perturbed line current magnitudes } \\
\text { and angles }\end{array}$ \\
\hline$C_{\mathrm{V} \delta}, C_{\theta \delta}$ & $\begin{array}{l}\text { bus voltage magnitude and angle output } \\
\text { matrices w.r.t. } \delta_{j}\end{array}$ \\
\hline $\boldsymbol{C}_{\mathrm{I}_{\mathrm{ft}} \delta}, \boldsymbol{C}_{\phi_{\mathrm{ft}} \delta}$ & $\begin{array}{l}\text { line current magnitude and angle output } \\
\text { matrices w.r.t. } \delta_{j}\end{array}$ \\
\hline$x_{d j}^{\prime}$ & transient reactance of the $j$-th machine \\
\hline$E_{\sim}^{\prime}$ & voltage behind the transient reactance \\
\hline$\tilde{E}_{j}^{\prime}$ & internal machine voltage \\
\hline$\tilde{\boldsymbol{Y}}$ & extended admittance matrix \\
\hline$\tilde{\boldsymbol{Y}}_{n n}^{-1} \tilde{\boldsymbol{Y}}_{n N}$ & bus voltage reconstruction matrix \\
\hline$\underset{\sim}{\tilde{\kappa}}=\kappa \angle \gamma$ & bus voltage reconstruction coefficient matrix \\
\hline$\tilde{\Psi}_{F T j}=$ & urrent reconstruction coefficient matr \\
\hline
\end{tabular}

$\boldsymbol{S}_{\mathrm{V}} \quad$ bus voltage magnitude mode shape

$\boldsymbol{S}_{\theta} \quad$ bus voltage angle modal mode shape

$\boldsymbol{S}_{I_{\mathrm{ft}}} \quad$ line current magnitude mode shape

$\boldsymbol{S}_{\phi_{\mathrm{ft}}} \quad$ line current angle mode shape

$\tau_{(p-q, k)} \quad$ time delay between Bus $p$ and Bus $q$ for the $k$-th mode

\section{References}

[1] J. H. Chow, G. Peponides, P. V. Kokotovic, B. Avramovic, and J. R. Winkelman, Time-Scale Modeling of Dynamic Networks with Applications to Power System, J. H. Chow, Ed. New York: Springer-Verlag, 1982.

[2] M. Klein, G. J. Rogers, and P. Kundur, "A Fundamental Study of Inter-Area Oscillations in Power Systems," IEEE Transactions on Power Systems, vol. 6, no. 3, pp. 914-921, Aug. 1991.

[3] G. Verghese, I. Perez-Arriga, and F. Schweppe, "Selective Modal Analysis with Application to Electric Power Systems, Part I: Heuristic Introduction, Part II: The Dynamic Stability Problem," IEEE Transactions on Power Apparatus and Systems, vol. PAS-101, pp. 3117-3134, 1982.

[4] P. Kundur, Power Systems Stability and Control. McGrawHill, 1994.

[5] G. Rogers, Power System Oscillations. MA: Kluwer, December 1999

[6] J.H. Chow, L. Vanfretti, et al, "Preliminary Synchronized Phasor Data Analysis of Disturbance Events in the US Eastern Interconnection," IEEE PES Power Systems Conference and Exposition, March 2009.

[7] L. Vanfretti, "Notions of phasor measurement-based power system model reduction of large power systems," M.Sc. Thesis, Rensselaer Polytechnic Institute, Troy, New York, July 2007.

[8] L. Vanfretti and J. Chow, "Computation and Analysis of Power System Voltage Oscillations from Interarea Modes," in IEEE PES General Meeting, July 2009, pp. 1-8.

[9] L. Vanfretti, T. M. L. Assis, J. H. Chow, L. Dosiek, J. Pierre, D. Trudnowski, and Y. Liu, "Data Analysis of the 2/26/08 Florida Disturbance," NASPI Work Group Meeting, Sacramento, CA, June 2009, available online: http://www.naspi.org/meetings/workgroup/workgroup.stm.

[10] FRCC Event Analysis Team, "FRCC System Disturbance and Underfrequency Load Sheeding Event Report, February 26, 2008 at 1:09 pm," Florida Reliability Coordinating Council, Tech. Rep., October 30, 2008.

[11] P. W. Sauer and M. A. Pai, Power System Dynamics and Stability. Upper Saddle River, New Jersey: Prentice Hall, 1998.

[12] L. Vanfretti, "Phasor measurement-based state estimation of electric power systems and linearized analysis of power system network oscillations," Ph.D Thesis, Rensselaer Polytechnic Institute, Troy, NY, December 2009.

[13] E. Z. Zhou, "Power oscillation flow study of electric power systems," Electrical Power \& Energy Systems, vol. 17, no. 2, pp. 143-150, 1995.

[14] J. Juang, Applied System Identification. New Jersey: PTR Prentice-Hall, 1994. 
[15] J. H. Chow and P. V. Kokotovic, "Time Scale Modeling of Sparse Dynamic Networks," IEEE Transactions on Automatic Control, vol. 30, no. 8, pp. 714-722, Aug. 1985.

[16] P. Anderson and A. Fouad, Power System Control and Stability. New York: Wiley-IEEE Press, 2002.

[17] J. H. Chow and K. W. Cheung, "A Toolbox for Power System Dynamics and Control Engineering Education and Research," IEEE Transactions on Power Systems, vol. 7, no. 4, pp. 1559-1564, Nov. 1992.

[18] E. V. Larsen and J. H. Chow, "SVC Control Design Concepts for System Dynamic Performance," IEEE Special Publication 87TH0187-5-PWR, Application of Static VAR Systems for System Dynamic Performance, 1987.

[19] E. V. Larsen, J. J. Sanchez-Gasca, and J. H. Chow, "Concepts for Design of FACTS Controllers to Damp Power Swings," IEEE Transactions on Power Systems, vol. 10, no. 2, pp. 948-956, May 1995.

\section{Biographies}

Luigi Vanfretti (M'03) is an Assistant Professor at the Electric Power Systems Division, School of Electrical Engineering, KTH Royal Institute of Technology, Stockholm, Sweden, where he was a PostDoctoral Research Fellow during May 2010.
He obtained the Electrical Engineering Degree (with a Power Engineering concentration) from Universidad de San Carlos de Guatemala in June 2005, and received the Francisco Vela award for outstanding academic achievement and best undergraduate thesis. During Fall 2005, he was a visiting researcher at the Department of Electronics and Electrical Engineering of The University of Glasgow, Scotland.

He received his MS in 2007 and $\mathrm{PhD}$ in 2009, both in Electric Power Engineering, from the Electrical, Computer, and Systems Engineering (ECSE) Department at Rensselaer Polytechnic Institute (RPI). During Spring 2010 he was a Post-Doctoral Research Associate at RPI working on synchrophasor applications. He was awarded the Charles M. Close Doctoral Prize from RPI in May, 2010 for his research and teaching work at ECSE.

His research interests are modeling, dynamics, stability and control of power systems; applications of PMU data for Smart Transmission Grids, and open source software for power system engineering.

Joe H. Chow(F'92) received his MS and PhD degrees from the University of Illinois, Urbana-Champaign. After working in the General Electric Power System business in Schenectady, he joined Rensselaer Polytechnic Institute in 1987. He is a professor of Electrical, Computer, and Systems Engineering and the Associate Dean of Engineering for Research and Graduate Programs. His research interests include multivariable control, power system dynamics and control, voltage-sourced converter-based FACTS Controllers, and synchronized phasor data. 


\section{Apprendix: Proof of the Network Sensitivity Properties}

In this appendix we give proofs for the expressions of the sensitivity properties in Section 4.4. For the bus voltage magnitude, the sensitivity properties are

$$
\begin{aligned}
& \sum_{j=1}^{N}\left(\frac{\partial V_{i}}{\partial \delta_{j}}\right)=0 \\
& \sum_{j=1}^{N}\left(\frac{\partial \theta_{i}}{\partial \delta_{j}}\right)=1
\end{aligned}
$$

Proof of property (61)

We obtain property (61) by adding the expressions in (19)

$$
\begin{aligned}
& \sum_{j=1}^{N}\left(\frac{\partial V_{i}}{\partial \delta_{j}}\right)=\left(\begin{array}{c}
-\sum_{\substack{p=1 \\
N-1}}^{N} \sum_{\substack{q=p+1 \\
N=1}}^{N} \kappa_{i p} \kappa_{i q} E_{p}^{\prime} E_{q}^{\prime} \sin \left(\delta_{p}+\gamma_{i p}-\delta_{q}-\gamma_{i q}\right) \\
+\sum_{\substack{p=1 \\
p \neq j}}^{N} \sum_{\substack{q \neq j \\
q \neq j}} \kappa_{i p} \kappa_{i q} E_{p}^{\prime} E_{q}^{\prime} \sin \left(\delta_{p}+\gamma_{i p}-\delta_{q}-\gamma_{i q}\right)
\end{array}\right)_{j=p}+
\end{aligned}
$$

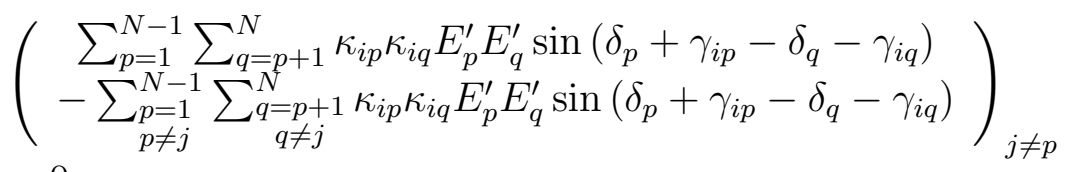

the elements in the first parenthesis of (63) cancel with the elements of the second parenthesis, thus, the sum of all bus voltage magnitude sensitivities at bus $i$ is zero.

Proof of property (62)

Property (62) is proved as follows. Replacing expression (22) in (62) results in

$$
\sum_{j=1}^{N}\left(\frac{\partial \theta_{i}}{\partial \delta_{j}}\right)=\frac{1}{\left|\tilde{V}_{i}\right|^{2}}\left(\sum_{j=1}^{N} \kappa_{i j}^{2} E_{j}^{\prime 2}+\sum_{j=1}^{N} \sum_{\substack{q=1 \\ q \neq j}}^{N} \kappa_{i j} \kappa_{i q} E_{j}^{\prime} E_{q}^{\prime} \cos \left(\delta_{j}+\gamma_{i j}-\delta_{q}-\gamma_{i q}\right)\right)
$$

Expanding the second summation term in (64)

$$
\begin{aligned}
& \sum_{j=1}^{N} \sum_{\substack{q=1 \\
q \neq j}}^{N} \kappa_{i j} \kappa_{i q} E_{j}^{\prime} E_{q}^{\prime} \cos \left(\delta_{j}+\gamma_{i j}-\delta_{q}-\gamma_{i q}\right)= \\
& \underbrace{\sum_{q=2}^{N} \kappa_{i 1} \kappa i q E_{1}^{\prime} E_{q}^{\prime} \cos \left(\delta_{1}+\gamma_{i 1}-\delta_{q}-\gamma_{i q}\right)}_{j=1} \\
& +\underbrace{\kappa_{i 2} \kappa_{i 1} E_{2}^{\prime} E_{1}^{\prime} \cos \left(\delta_{2}+\gamma_{i 2}-\delta_{1}-\gamma_{i 1}\right)+\sum_{q=3}^{N} \kappa_{i 2} \kappa_{i q} E_{2}^{\prime} E_{q}^{\prime} \cos \left(\delta_{2}+\gamma_{i 2}-\delta_{q}-\gamma_{i q}\right)}_{j=2} \\
& +\underbrace{\begin{array}{r}
\kappa_{i 3} \kappa_{i 1} E_{3}^{\prime} E_{1}^{\prime} \cos \left(\delta_{3}+\gamma_{i 3}-\delta_{1}-\gamma_{i 1}\right)+\kappa_{i 3} \kappa_{i 2} E_{3}^{\prime} E_{2}^{\prime} \cos \left(\delta_{3}+\gamma_{i 3}-\delta_{2}-\gamma_{i 2}\right)+ \\
\sum_{q=4}^{N} \kappa_{i 3} \kappa_{i q} E_{3}^{\prime} E_{q}^{\prime} \cos \left(\delta_{3}+\gamma_{i 3}-\delta_{q}-\gamma_{i q}\right)
\end{array}}_{j=3} \\
& +\ldots \\
& +\sum_{p=1}^{N-2} \kappa_{i(N-1)} \kappa_{i p} E_{(N-1)}^{\prime} E_{p}^{\prime} \cos \left(\delta_{(N-1)}+\gamma_{i(N-1)}-\delta_{N}-\gamma_{i N}\right)
\end{aligned}
$$

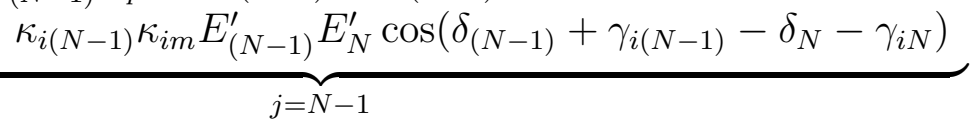

$$
\begin{aligned}
& +\underbrace{\sum_{p=1}^{N-1} \kappa_{i N} \kappa_{i p} E_{N}^{\prime} E_{p}^{\prime} \cos \left(\delta_{N}+\gamma_{i N}-\delta_{p}-\gamma_{i p}\right)}_{j=N}
\end{aligned}
$$


Grouping all the summation terms in (65), except the $j=N$ term, yields

$$
\begin{aligned}
& \underbrace{\sum_{q=2}^{N} \kappa_{i 1} \kappa i q E_{1}^{\prime} E_{q}^{\prime} \cos \left(\delta_{1}+\gamma_{i 1}-\delta_{q}-\gamma_{i q}\right)}_{j=1}+\underbrace{\sum_{q=3}^{N} \kappa_{i 2} \kappa_{i q} E_{2}^{\prime} E_{q}^{\prime} \cos \left(\delta_{2}+\gamma_{i 2}-\delta_{q}-\gamma_{i q}\right)}_{j=2}+ \\
& \underbrace{\sum_{q=4}^{N} \kappa_{i 3} \kappa_{i q} E_{3}^{\prime} E_{q}^{\prime} \cos \left(\delta_{3}+\gamma_{i 3}-\delta_{q}-\gamma_{i q}\right)}_{j=3}+\ldots+\underbrace{\sum_{q=N}^{N} \kappa_{i(N-1)} \kappa_{i q} E_{(N-1)}^{\prime} E_{q}^{\prime} \cos \left(\delta_{(N-1)}+\gamma_{i(N-1)}-\delta_{q}-\gamma_{i q}\right)}_{j=N-1}
\end{aligned}
$$$$
=\sum_{p=1}^{(N-1)} \sum_{q=p+1}^{N} \kappa_{i p} \kappa_{i q} E_{p}^{\prime} E_{1}^{\prime} \cos \left(\delta_{p}+\gamma_{i p}-\delta_{q}-\gamma_{i q}\right)
$$

Next, we group all the other terms in (65) including the summation term for $j=N$,

$$
\begin{aligned}
& \underbrace{0}_{j=1}+\underbrace{\kappa_{i 2} \kappa_{i 1} E_{2}^{\prime} E_{1}^{\prime} \cos \left(\delta_{2}+\gamma_{i 2}-\delta_{2}-\gamma_{i 1}\right)}_{j=2}+ \\
& \underbrace{\kappa_{i 3} \kappa_{i 1} E_{3}^{\prime} E_{1}^{\prime} \cos \left(\delta_{3}+\gamma_{i 3}-\delta_{1}-\gamma_{i 1}\right)+\kappa_{i 3} \kappa_{i 2} E_{3}^{\prime} E_{2}^{\prime} \cos \left(\delta_{3}+\gamma_{i 3}-\delta_{2}-\gamma_{i 2}\right)}_{j=3}+ \\
& \quad \ldots \quad+ \\
& \underbrace{\sum_{p=1}^{N-2} \kappa_{i(N-1)} \kappa_{i p} E_{(N-1)}^{\prime} E_{p}^{\prime} \cos \left(\delta_{(N-1)}+\gamma_{i(N-1)}-\delta_{N}-\gamma_{i N}\right)}_{j=N-1}+\underbrace{\sum_{p=1}^{N-1} \kappa_{i m} \kappa_{i p} E_{N}^{\prime} E_{p}^{\prime} \cos \left(\delta_{N}+\gamma_{i N}-\delta_{p}-\gamma_{i p}\right)}_{j=N}
\end{aligned}
$$$$
=\sum_{p=1}^{(N-1)} \sum_{q=p+1}^{N} \kappa_{i p} \kappa_{i q} E_{p}^{\prime} E_{1}^{\prime} \cos \left(\delta_{p}+\gamma_{i p}-\delta_{q}-\gamma_{i q}\right)
$$

Expressions (66) and (67) can be used to rewrite (64) as

$$
\sum_{j=1}^{N}\left(\frac{\partial \theta_{i}}{\partial \delta_{j}}\right)=\frac{1}{\left|\tilde{V}_{i}\right|^{2}}\left(\sum_{j=1}^{N} \kappa_{i j}^{2} E_{j}^{\prime 2}+2 \sum \sum_{p=1}^{N-1} \sum_{q=p+1}^{N} \kappa_{i p} \kappa_{i q} E_{p}^{\prime} E_{q}^{\prime} \cos \left(\delta_{p}+\gamma_{i p}-\delta_{q}-\gamma_{i q}\right)\right)
$$

The $V_{i}$ expression in (15) can be rewritten in rectangular form as follows

$$
\tilde{V}_{i}=\sum_{j=1}^{N} \kappa_{i j} E_{j}^{\prime} \cos \left(\delta_{j}+\gamma_{i j}\right)+j \sum_{j=1}^{N} \kappa_{i j} E_{j}^{\prime} \sin \left(\delta_{j}+\gamma_{i j}\right)
$$

using (69) we derive an expression for the bus voltage magnitude

$$
V_{i}=\sqrt{\left(\sum_{j=1}^{N} \kappa_{i j} E_{j}^{\prime} \cos \left(\delta_{j}+\gamma_{i j}\right)\right)^{2}+\left(\sum_{j=1}^{N} \kappa_{i j} E_{j}^{\prime} \sin \left(\delta_{j}+\gamma_{i j}\right)\right)^{2}}
$$

To expand the two terms inside (70) we use the following identity

$$
\left(\sum_{k=1}^{n} a_{k} b_{k}\right)^{2}=\sum_{k=1}^{n} a_{k} b_{k}+2 \sum_{i=1}^{n-1} \sum_{j=i+1}^{n} a_{i} b_{i} a_{j} b_{j}
$$

The expanded form of the first term in the (70) is

$$
\begin{aligned}
& \left(\sum_{k=1}^{N} \kappa_{i j} E_{j}^{\prime} \cos \left(\delta_{j}+\gamma_{i j}\right)\right)^{2}= \\
& \sum_{j=1}^{N} \kappa_{i j}^{2} E_{j}^{2} \cos ^{2}\left(\delta_{j}+\gamma_{i j}\right)+2 \sum_{p=1}^{N-1} \sum_{q=p+1}^{N} \kappa_{i p} \kappa_{i q} E_{p}^{\prime} E_{q}^{\prime} \cos \left(\delta_{p}+\gamma_{i p}\right) \cos \left(\delta_{q}+\gamma_{i q}\right)
\end{aligned}
$$


and expanding the second term is

$$
\begin{aligned}
& \left(\sum_{k=1}^{N} \kappa_{i j} E_{j}^{\prime} \sin \left(\delta_{j}+\gamma_{i j}\right)\right)^{2}= \\
& \sum_{j=1}^{N} \kappa_{i j}^{2} E_{j}^{2} \sin ^{2}\left(\delta_{j}+\gamma_{i j}\right)+2 \sum_{p=1}^{N-1} \sum_{q=p+1}^{N} \kappa_{i p} \kappa_{i q} E_{p}^{\prime} E_{q}^{\prime} \sin \left(\delta_{p}+\gamma_{i p}\right) \sin \left(\delta_{q}+\gamma_{i q}\right)
\end{aligned}
$$

Adding (72) and (73), and using the identity

$$
\cos \theta_{1} \cos \theta_{2}+\sin \theta_{1} \sin \theta_{2}=\cos \left(\theta_{1}-\theta_{2}\right)
$$

we obtain an expression for the voltage magnitude

$$
V_{i}=\left(\sum_{j=1}^{N} \kappa_{i j}^{2} E_{j}^{2}+2 \sum_{p=1}^{N-1} \sum_{q=p+1}^{N} \kappa_{i p} \kappa_{i q} E_{p}^{\prime} E_{q}^{\prime} \cos \left(\delta_{p}+\gamma_{i p}-\delta_{1}-\gamma_{i q}\right)\right)^{1 / 2}
$$

From (74) we note that the expression inside the parenthesis in the left hand side of (68) corresponds to $\left|\tilde{V}_{i}\right|^{2}$, thus we conclude that the property for the the bus voltage angle sensitivities is

$$
\sum_{j=1}^{N}\left(\frac{\partial \theta_{i}}{\partial \delta_{j}}\right)=1
$$

The line current sensitivity properties given in (48) follow the same proof as shown above for the bus voltage magnitude and bus voltage angle, and thus, are not shown here. 